\title{
Conventional Magnetic Superconductors
}

\author{
C. T. Wolowiec ${ }^{\mathrm{a}, \mathrm{b}}$, B. D. White ${ }^{\mathrm{a}, \mathrm{b}}$, M. B. Maple $\mathrm{a}^{\mathrm{a}, \mathrm{b}, *}$ \\ ${ }^{a}$ Department of Physics, University of California, San Diego, La Jolla, California 92093, USA \\ ${ }^{b}$ Center of Advanced Nanoscience, University of California, San Diego, La Jolla, California 92093, USA
}

\begin{abstract}
We discuss several classes of conventional magnetic superconductors including the ternary rhodium borides and molybdenum chalcogenides (or Chevrel phases), and the quaternary nickel-borocarbides. These materials exhibit some exotic phenomena related to the interplay between superconductivity and long-range magnetic order including: the coexistence of superconductivity and antiferromagnetic order; reentrant and double reentrant superconductivity, magnetic field induced superconductivity, and the formation of a sinusoidally-modulated magnetic state that coexists with superconductivity. We introduce the article with a discussion of the binary and pseudobinary superconducting materials containing magnetic impurities which at best exhibit short-range "glassy" magnetic order. Early experiments on these materials led to the idea of a magnetic exchange interaction between the localized spins of magnetic impurity ions and the spins of the conduction electrons which plays an important role in understanding conventional magnetic superconductors. These advances provide a natural foundation for investigating unconventional superconductivity in heavy-fermion compounds, cuprates, and other classes of materials in which superconductivity coexists with, or is in proximity to, a magnetically-ordered phase.
\end{abstract}

Keywords: Superconductivity, magnetism, exchange interaction, antiferromagnetic order, ferromagnetic order, Kondo effect, pair-breaking, paramagnetic impurity, rare earth ion

\section{Introduction}

In 1957, V. L. Ginzburg was the first to theoretically address the question of whether superconductivity could occur in a ferromagnetic material [1]. The first empirical investigations of the interplay between magnetism and superconductivity were performed by Matthias, Suhl, and Corenzwit in 1958 who substituted small quantities of magnetic RE impurities into the superconducting element lanthanum and observed a rapid and linear suppression of $T_{c}$ with increasing impurity concentration $[2,3]$. The exchange interaction between the paramagnetic impurity spins and the conduction electrons spins was proposed to account for the significant reduction of $T_{c}$. These experiments and theoretical advances $[4,5,6]$ led to the discovery of novel magnetic superconducting compounds which exhibited exotic phenomena arising from the interplay between superconductivity and long-range magnetic order.

This article will attempt to highlight some of the interesting properties for the following paramagnetic and magnetic superconducting materials: (1) superconducting elements and binary compounds containing small amounts of paramagnetic impurities (transition metal (T), rare earth (RE), or actinide (A) ions with partially-filled $d$ - or $f$-electron shells) and (2) superconducting compounds with an ordered sublattice of RE ions that exhibit long-range magnetic order which were first observed in the ternary rhodium borides and molybdenum chalcogenides (or Chevrel phases) discovered in the 1970s [7, 8, 9] and then

*Corresponding Author: mbmaple@ucsd.edu later in the quaternary borocarbides discovered in the $1990 \mathrm{~s}$ $[10,11,12,13,14,15]$.

\section{Binary and Pseudobinary Compounds: Paramagnetic Impurities in Superconductors}

In the first part of this article, we discuss some of the interesting properties observed in binary and pseudobinary superconducting compounds containing dilute concentrations of paramagnetic impurities. Examples of binary systems discussed include: $\mathrm{La}_{1-x} \mathrm{RE}_{x}, \mathrm{Th}_{1-x} \mathrm{Gd}_{x}, \mathrm{~Pb}_{1-x} \mathrm{Gd}_{x}$, and $\mathrm{Th}_{1-x} \mathrm{U}_{x}$; examples of pseudobinary systems discussed include: $\mathrm{La}_{1-x} \mathrm{Gd}_{x} \mathrm{Al}_{2}$, $\mathrm{La}_{3-x} \mathrm{Ce}_{x} \mathrm{In}$, and $\left(\mathrm{La}_{1-y} \mathrm{Th}_{y}\right)_{1-x} \mathrm{Ce}_{x}$. Hybridization between the $d$ - or $f$-electron states of the substituted magnetic ions and the conduction electron states produce a variety of exotic phenomena including: the exchange interaction as a magnetic pairbreaking mechanism that results in a rapid suppression of superconductivity; gapless superconductivity in compounds containing the requisite levels of impurity concentrations; and the many-body physics related to the Kondo-effect which can compete with superconductivity and also give rise to interesting phenomena such as reentrant superconductivity and temperature dependent pair-breaking. The theoretical and experimental advances made in studying these binary and pseudobinary superconducting compounds led to the discovery of magnetically ordered superconductors and are important for understanding the interplay between long-range magnetic order and superconductivity in the ternary rhodium borides, Chevrel phases, and quaternary borocarbides discussed later in this article. 


\subsection{Ferromagnetic Exchange Interaction}

A nearly linear and rapid depression of the superconducting transition temperature $T_{c}$ with increasing concentration of RE impurity ion is displayed in Fig. 1 for the case of Gd impurities dissolved into the face-centered cubic (fcc) allotrope of elemental lanthanum $\left(T_{c 0}=6 \mathrm{~K}\right)$. The precipitous drop in $T_{c}$ with increasing $\mathrm{Gd}$ concentration $x$ precludes any co-existence of superconductivity and magnetic order; superconductivity is all but extinct at a much lower concentration of $x \sim 1 \%$ before magnetic order (which is best characterized in this study as short range and extends over only several lattice spacings) begins to set in at $x \sim 2.5 \%\left(T_{M} \simeq 1 \mathrm{~K}\right)$. Matthias et al. observed that the suppression of $T_{c}$ correlates with the spin, rather than the magnetic moment, of the RE ions and proposed a magnetic exchange interaction between the spin of the RE ions and the local spin density of the conduction electrons within the host superconductor as a pair-breaking mechanism to account for the rapid suppression of $T_{c}$ [2].

The exchange Hamiltonian between the magnetic moment of the RE impurity ion and the spin of the electron is given as:

$$
H_{e x}=-2 \mathcal{J}\left(g_{J}-1\right) \boldsymbol{J} \cdot \boldsymbol{s},
$$

where $g_{J}$ is the Landè g-factor for the Hund's rule ground state of the RE ion [19]. The exchange interaction parameter $\mathcal{J}$ that appears in Equation 1 is generally expressed as the sum of two terms: $\mathcal{J} \simeq \mathcal{J}_{0}+\mathcal{J}_{1}$. The ferromagnetic term $\mathcal{J}_{0}$ is positive $\left(\mathcal{J}_{0}>0\right)$ which favors parallel alignment between the impurity spin and the conduction electron spin density at the impurity site, while $\mathcal{J}_{1}$ is an antiferromagnetic term $\left(\mathcal{J}_{1}<0\right)$ that favors antiparallel alignment. The degree to which the $d$ - or $f$-electrons hybridize with the conduction electrons determines whether $\mathcal{J}_{0}$ or $\mathcal{J}_{1}$ will be dominant. For transition metal impurities with partially filled $d$-electron shells, the orbital moment $\boldsymbol{L}$ is quenched, and the exchange Hamiltonian has the form:

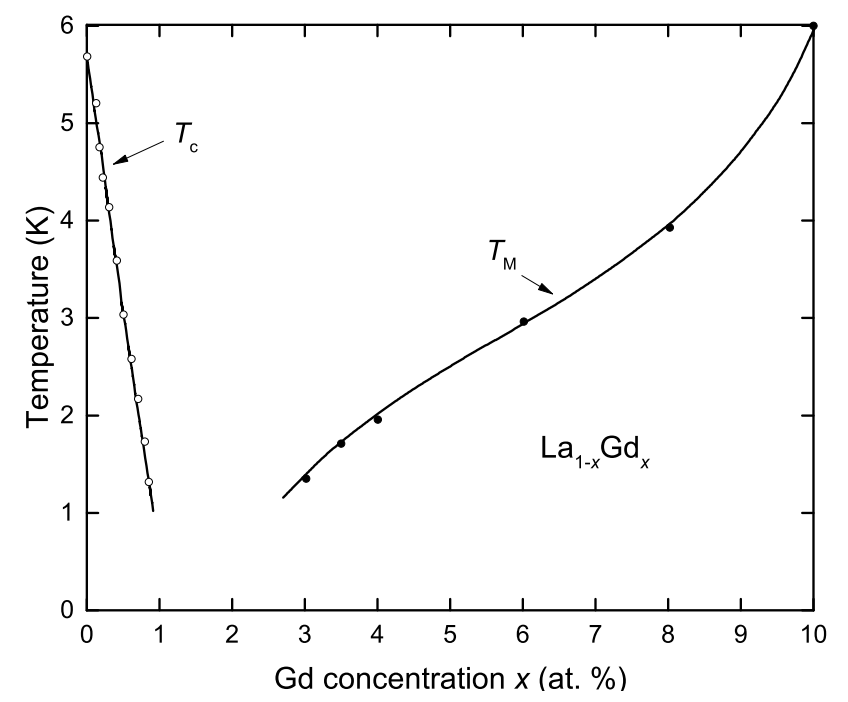

Figure 1: Superconducting transition temperature $T_{c}$ and magnetic ordering temperature $T_{M}$ vs. Gd concentration for the $\mathrm{La}_{1-x} \mathrm{Gd}_{x}$ system. (from Ref. [2])

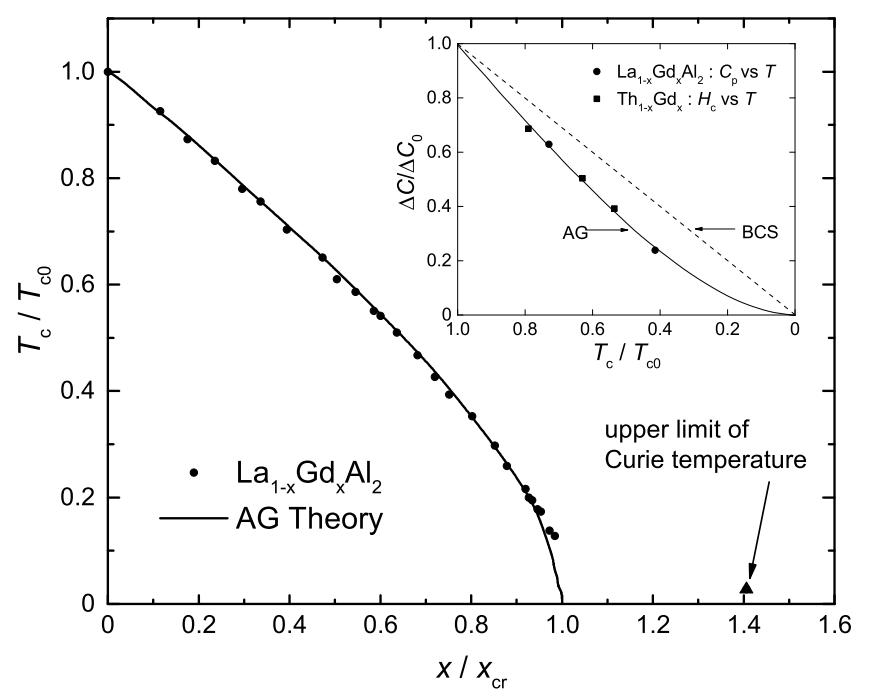

Figure 2: Reduced superconducting transition temperature $T_{c} / T_{c 0}$ vs. reduced Gd concentration $x / x_{c r}$ for the $\mathrm{La}_{1-x} \mathrm{Gd}_{x} \mathrm{Al}_{2}$ system compared to the Abrikosov-Gor'kov (AG) theory (solid curve). The value of the superconducting critical temperature at $x=0$ is $T_{c 0}=3.24 \mathrm{~K}$ and the value of the critical Gd concentration is $x_{c r}=0.590$ at. \%. The reduced Curie-Weiss temperature $\Theta / T_{C}$ measured at $x / x_{c r}=1.41$ is denoted by the solid triangle. (from Ref. [5, 16]) Inset: Reduced specific heat jump $\Delta C / \Delta C_{0}$ vs. reduced transition temperature $T_{c} / T_{c 0}$ for the $\mathrm{La}_{1-x} \mathrm{Gd}_{x} \mathrm{Al}_{2}$ and $\mathrm{Th}_{1-x} \mathrm{Gd}_{x}$ systems. The dashed line represents the BCS law of corresponding states [17] whereas the solid line is based on AG theory as calculated by Skalski et al. [18]. (from Ref. [6])

$$
H_{e x}=-2 \mathcal{J} S \cdot s,
$$

where $S$ is the spin of the transition metal impurity ion.

In the case of moderate hybridization between the localized $d$ - or $f$ - electron and conduction electron states, the exchange interaction is dominated by the antiferromagnetic term $\mathcal{J}_{1}$. In this case, th localized magnetic moment of the impurity ion is quenched (or screened out) by the spin polarization of the surrounding conduction electron density via the Kondo effect. In the case of weak hybridization, the exchange interaction parameter $\mathcal{J}$ is dominated by the ferromagnetic (Heisenberg) exchange term $\mathcal{J}_{0}$. Drastic differences in the superconducting properties arise from the subtle differences between weak and moderate hybridization in superconductors containing paramagnetic impurities. In the case of strong hybridization, the exchange interaction does not appear to provide an appropriate description of the physics; however, other pair-breaking or pair weakening mechanisms can still lead to significant effects on $T_{c}$ [6].

\subsubsection{Experiment and the Abrikosov-Gor'kov (AG) Theory}

Rare earth (RE) impurities with stable valences that tend to exhibit ferromagnetic exchange interactions when embedded in a host superconductor include: $\mathrm{Pr}, \mathrm{Nd}, \mathrm{Gd}, \mathrm{Tb}, \mathrm{Dy}, \mathrm{Ho}$, and Er. These impurities can drastically affect the superconducting properties of their host materials [2]. This is well illustrated by the suppression of $T_{c}$ with increasing Gd concentration $x$ in the $\mathrm{La}_{1-x} \mathrm{Gd}_{x} \mathrm{Al}_{2}$ system $[2,20]$. The rapid suppression of $T_{c}$ with $x$ is evident from the curve of $T_{c} / T_{c 0}$ vs. $x / x_{c r}$ for the 


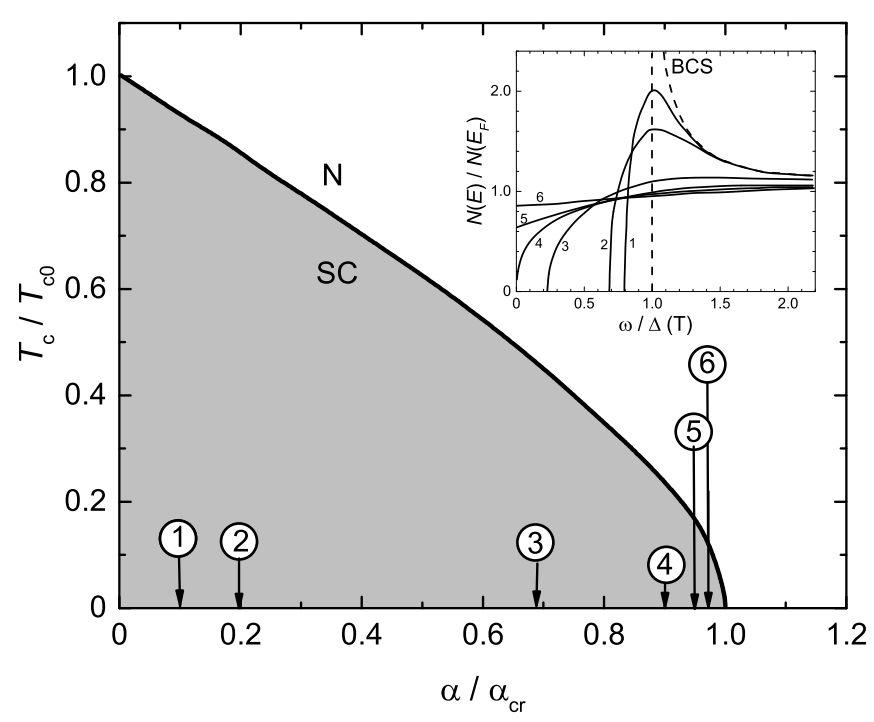

Figure 3: Universal relation between the reduced superconducting transition temperature $T_{c} / T_{c 0}$ and the reduced pair-breaking parameter $\alpha / \alpha_{c r}$. Inset: Normalized density of states $N(E) / N\left(E_{F}\right)$ vs. normalized energy $\omega / \Delta$ curves $(T=$ $0 \mathrm{~K})$ corresponding to different values of the reduced pair-breaking parameter $\alpha / \alpha_{c r}$. (from Ref. [21])

$\mathrm{La}_{1-x} \mathrm{Gd}_{x} \mathrm{Al}_{2}$ system as shown in Fig. 2 (where $x_{c r}$ is the critical concentration of $\mathrm{Gd}$ impurities above which superconductivity does not appear at any temperature) [2]. The curve of $T_{c} / T_{c 0} \mathrm{vs}$. $x / x_{c r}$ is well described by a universal function that is calculated using the Abrikosov-Gor'kov (hereafter AG) theory of paramagnetic impurities in superconductors [4]. The results, which are displayed in the main panel of Fig. 2, are in good agreement with AG theory up to concentrations very near $x_{c r}$. The effect on $T_{c}$ in this system is largest when the interactions between the Gd impurities are sufficiently weak so that they may be neglected over a considerable temperature range below $T_{c 0}$. Furthermore, because the Gd impurity is a half filled $S$-state ion, it is insensitive to the effects of the crystalline electric field (CEF) on the Hund's rule ground state.

The AG theory addresses the superconducting properties in the presence of impurity spins by introducing the pair-breaking parameter $\alpha$ :

$$
\alpha \equiv \frac{1}{\tau_{s c}}=\frac{x}{\hbar} N\left(E_{F}\right) \mathcal{J}^{2} S(S+1),
$$

where $x$ is the paramagnetic impurity concentration and $N\left(E_{F}\right)$ is the density of states (for one spin direction) at the Fermi level. For RE impurities, the spin of the paramagnetic impurity $\boldsymbol{S}$ is replaced by its projection onto the total angular momentum vector $\boldsymbol{J}=\boldsymbol{L}+\boldsymbol{S}$ for the Hund's rule ground state. Hence, the AG pair-breaking parameter $\alpha$ becomes:

$$
\alpha=\frac{x}{\hbar} N\left(E_{F}\right) \mathcal{J}^{2}\left(g_{J}-1\right)^{2} J(J+1) .
$$

The important characteristics of the pair-breaking parameter $\alpha$ are: (1) it is proportional to the impurity concentration $x$, (2) it is proportional to $\mathcal{J}^{2}$ so it depends only on the magnitude of the exchange interaction parameter $\mathcal{J},(3)$ it is proportional to

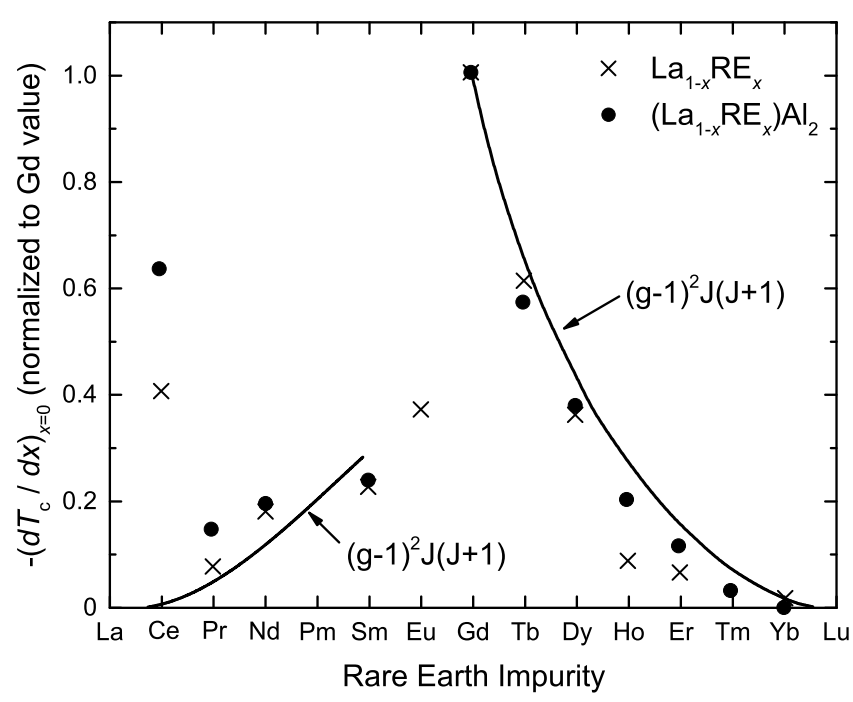

Figure 4: Initial rates of depression of $T_{c}$ with rare earth (RE) impurity concentration $\left(-d T_{c} / d x\right)_{x=0}$ vs. RE impurity for the $\mathrm{La}_{1-x} \mathrm{RE}_{x}$ and $\mathrm{La}_{1-x} \mathrm{RE}_{x} \mathrm{Al}_{2}$ alloys. The initial suppression rates for each $\mathrm{RE}$ are normalized to the $\mathrm{Gd}$ suppression rates of $5.3 \mathrm{~K}$ per at. $\%$ and $3.79 \mathrm{~K}$ per at. $\%$ for the $\mathrm{La}_{1-x} \mathrm{Gd}_{x}$ and $\mathrm{La}_{1-x} \mathrm{Gd}_{x} \mathrm{Al}_{2}$ systems, respectively. The solid lines correspond to the de Gennes factor $\left(g_{J}-1\right)^{2} J(J+1)$ vs. RE (normalized to the Gd value). (from Ref. [5])

$S(S+1)$ for transition metal solutes where $L$ is quenched or to the de Gennes factor $\left(g_{J}-1\right)^{2} J(J+1)$ for RE solutes, and (4) it is independent of temperature.

AG theory predicts a second order phase transition from the normal to the superconducting state and a subsequent rapid decrease in the transition temperature $T_{c}$ as a function of the pair-breaking parameter $\alpha$ given by the universal relation:

$$
\ln \left(\frac{T_{c}}{T_{c 0}}\right)=\Psi\left(\frac{1}{2}\right)-\Psi\left(\frac{1}{2}+0.14 \frac{\alpha}{\alpha_{c r}} \frac{T_{c 0}}{T_{c}}\right) .
$$

The universal relation between $T_{c} / T_{c 0}$ and $\alpha / \alpha_{c r}$ is displayed in the main panel of Fig. 3. The reduced pair-breaking parameter $\alpha / \alpha_{c r}$ may be replaced with the reduced concentration $x / x_{c r}$ where $x_{c r}$ is the critical value of the impurity concentration that completely destroys superconductivity. The reduced transition temperature $T_{c} / T_{c 0}$ will also follow a universal function of the reduced impurity concentration $x / x_{c r}$ :

$$
\ln \left(\frac{T_{c}}{T_{c 0}}\right)=\Psi\left(\frac{1}{2}\right)-\Psi\left(\frac{1}{2}+0.14 \frac{x}{x_{c r}} \frac{T_{c 0}}{T_{c}}\right),
$$

and in the dilute concentration limit $(x \rightarrow 0)$, Equation 6 has the linear asymptotic form:

$$
\frac{T_{c}}{T_{c 0}}=1-0.691 \frac{x}{x_{c r}} .
$$

Thus, in the low impurity concentration limit, the AG theory correctly predicts the initial linear depression of $T_{c}$ for $\mathrm{Gd}$ substitution into superconducting $\mathrm{LaAl}_{2}$ as shown in Fig. 2. The initial suppression rate of $T_{c}$ with $x$ is then given by the expression:

$$
\left.\frac{d T_{c}}{d x}\right|_{x=0}=-\frac{\pi^{2}}{2} \frac{N\left(E_{F}\right)}{k_{B}} \mathcal{J}^{2}\left(g_{J}-1\right)^{2} J(J+1) .
$$


The initial suppression rates of $T_{c}$ with RE impurity concentration $\left(d T_{c} / d x\right)_{x=0}$ are plotted against the RE in Fig. 4, which clearly shows that the depression of the superconducting transition temperature $\Delta T_{c}=T_{c}-T_{c 0}$ with RE impurity concentration $\Delta x$ is approximately proportional to the de Gennes factor $\left(g_{J}-1\right)^{2} J(J+1)$. Hence, $\left(d T_{c} / d x\right)_{x=0}$ exhibits a characteristic variation with RE solute ion and is a maximum for the halffilled $4 f$-electron shell of $\mathrm{RE}=\operatorname{Gd}[2,22,23]$. The data displayed in Fig. 4 also reveal that $\left(d T_{c} / d x\right)_{x=0}$ values generally lie above (below) the de Gennes factor for the lighter (heavier) $\mathrm{RE}$ ions to the left (right) of $\mathrm{Gd}$ in the periodic table. This behavior appears to be a general property that may be attributed to both a general decrease in $|\mathcal{J}|$ with increasing RE atomic number and the splitting of the RE ion energy levels due to the CEF, which is generally larger for the lighter RE ions [24]. The anomalously large value for the initial rate of depression of $T_{c}$ observed for Ce impurities in the $\mathrm{La}$ and $\mathrm{LaAl}_{2}$ superconducting hosts can be explained as a consequence of a large negative contribution to the exchange interaction and the Kondo effect which will be addressed below in Section 2.2. Large depressions of $T_{c}$ for Ce impurities have been observed in other systems (e.g., $\mathrm{Y}_{1-x} \mathrm{Ce}_{x} \mathrm{~B}_{6}$ [25]) and for other RE impurities, in which the RE ions have valence instabilities (e.g., $\mathrm{La}_{1-x} \mathrm{Sm}_{x} \mathrm{Sn}_{3}$ [26]).

In addition to its ability to explain the rapid linear decrease in $T_{c}$ with increasing $x$ (albeit in the low concentration limit of $x \sim 1$ at. \%), the AG theory successfully accounts for the dependence of the reduced jump in the specific heat $\Delta C$ at $T_{c}$ in a superconductor containing low concentrations of paramagnetic impurities. The data for the reduced specific heat jump $\Delta C / \Delta C_{0}$ vs. the reduced transition temperature $T_{c} / T_{c 0}$ for both the $\mathrm{La}_{1-x} \mathrm{Gd}_{x} \mathrm{Al}_{2}$ [27] and $\mathrm{Th}_{1-x} \mathrm{Gd}_{x}$ [28] systems are shown in the inset of Fig. 2. Here, $\Delta C_{0}$ corresponds to the specific heat jump of the superconductor in the absence of impurities. The specific heat behavior of both systems deviates markedly from the BCS law of corresponding states, $\Delta C / \Delta C_{0}=T_{c} / T_{c 0}$ [17], but are in good agreement with the universal relation $\Delta C / \Delta C_{0}$ $=\mathcal{U}\left(T_{c} / T_{c 0}\right)$ predicted by AG theory (which is indicated by the solid curve determined from calculations by Skalski et al.) [18].

\subsubsection{Gapless Superconductivity}

Perhaps the most striking prediction of the AG theory is the phenomenon of "gapless superconductivity". Abrikosov and Gor'kov predicted a value of the paramagnetic impurity concentration $x=0.91 x_{c r}$ for which the gap in the energy spectrum vanishes at all temperatures [4]. An increase in $x$ corresponds to an increase in $\alpha$ which in turn implies a decrease in the lifetime $\tau_{s c}$ of the superconducting electron pairs (see Equation 3). This results in broadening of the available states in the superconducting phase based on the well known energy uncertainty relation $\Gamma \sim h / \tau$. Hence, as the value of the pair-breaking parameter $\alpha$ increases, the density of states curves shown in the inset of Fig. 3 deviate from the divergence occurring at the energy value $\omega=\Delta$ as predicted by the BCS theory (where $\Delta$ is the BCS value of the energy gap in the absence of impurities at $T=$ $0 \mathrm{~K}$ ). The broadening in energy of the density of states curves introduces states with energy levels within the energy gap as displayed in the inset of Fig. 3 where the numbers 1 through 6 denote the density of states curves that correspond to the values of $\alpha / \alpha_{c r}$ labeled by the same numbers in the main panel of Fig. 3. The energy gap just closes for the curve labeled number 4 corresponding to $\alpha / \alpha_{c r}=0.91$. As a consequence, the energy gap for a superconductor containing paramagnetic impurities $\Omega_{G}(\alpha)$ deviates from the BCS calculation of the energy gap, $\Delta(\alpha)$, such that $\Omega_{G}(\alpha) \rightarrow 0$ meV faster than the superconducting transition temperature $T_{c} \rightarrow 0 \mathrm{~K}$. For temperatures sufficiently close to $T_{c}$, the superconductor is gapless for all concentrations $0 \leq x \leq x_{c r}$, whereas for concentrations in the range $0.91 x_{c r} \leq$ $x \leq x_{c r}$, the superconductor is gapless at all temperatures.

Gapless superconductivity was observed in a series of experiments performed in the 1960s [29, 30, 31]. In 1962, Reif and Woolf reported on tunneling experiments performed on thin films of the $\mathrm{Pb}_{1-x} \mathrm{Gd}_{x}$ system in which they observed the energy gap vanish, but at a value of the impurity concentration $x \sim$ $0.5 x_{c r}$ (much lower than $x=0.91 x_{c r}$ as predicted by the AG theory) [29]. The discrepancy has been attributed to the tendency toward ferromagnetic ordering of the Gd impurity spins at low temperatures $[32,33]$. Specific heat measurements performed on the $\mathrm{La}_{1-x} \mathrm{Gd}_{x}$ system by Finnemore et al. in 1965 showed that an increase in $\mathrm{Gd}$ concentration results in a decrease in the rate at which the specific heat $C / \gamma T_{c}$ approaches zero which may be attributed to a diminishing energy gap $\Omega_{G}(\alpha)$; however, they could not rule out the possibility that the effect on the rate of decrease in the specific heat $C$ with $T_{c}$ was due instead to magnetic interactions among the Gd impurity ions [31].

\subsubsection{Departures from $A G$ Theory: $C e_{1-x} G d_{x} R u_{2}$}

Experiments by Matthias and coworkers on the $\mathrm{Ce}_{1-x} \mathrm{Gd}_{x} \mathrm{Ru}_{2}$ system revealed that the initial depression of $T_{c}$ was not rapid, in contrast to the prediction of AG theory. Instead, there was a slight enhancement of $T_{c}$ up to $x \sim 1 \%$ which was followed by a relatively weak depression of $T_{c}$ at higher concentrations. This is probably due to the combination of the change in conduction electron concentration as trivalent Gd is substituted for tetravalent $\mathrm{Ce}$ and a relatively weak exchange interaction. As a result, there is a substantial region (namely $1 \% \leq x \leq 6 \%$ ) over which the $T_{c}$ vs. $x$ curve overlaps the magnetic ordering temperature $T_{M}$ vs. $x$ curve [3].

Most investigations of superconductor-impurity systems in the dilute limit reveal that the experimental $T_{c}(x)$ curves are in agreement with AG theory up to reduced concentrations $x / x_{c r}$ $\sim 0.7$ (corresponding to $T_{c} / T_{c 0} \sim 0.45$ ). However, at higher concentrations, there are striking departures of $T_{c}(x)$ from the universal functions based on the AG theory. This observation underscores that AG theory is suitable only in the range of impurity concentration where interactions between the magnetic moments of the solute ions are sufficiently weak and under conditions which favor weak hybridization between the localized $f$ electrons of the impurity ion and the conduction electrons.

\subsection{Antiferromagnetic Exchange Interaction and the Kondo Effect}

RE impurities with unstable valence configurations such as $\mathrm{Ce}, \operatorname{Pr}$ (occasionally), Sm, Eu, Tm, and Yb (along with the tran- 
sition metal ions with partially-filled $d$-electron shells and actinide ions with partially-filled $f$-electron shells) tend to exhibit a moderate amount of mixing of the localized $d$ - or $f$-electron states of the impurity ion with the conduction electron states. This is represented by the matrix elements $\left\langle V_{k l}^{2}\right\rangle$ in the FriedelAnderson Hamiltonian [34, 35]. According to Schrieffer and Wolff [36], these matrix elements can be transformed into an equivalent form that appears in an exchange model as an antiferromagnetic exchange interaction term $\mathcal{J}_{1}$ :

$$
\mathcal{J}_{1} \sim-\frac{\left\langle V_{k l}^{2}\right\rangle}{E_{l}}<0,
$$

where $E_{l}$ is the energy separating the localized impurity state and the Fermi energy. The energetics of a negative $(\mathcal{J}<0)$ exchange interaction, $H_{e x}=-2 \mathcal{J}\left(g_{J}-1\right) S \cdot s$, dictate an antiparallel orientation of the RE impurity spin $S$ and the spin polarization $s$ of the local conduction electron density. The situation leads to the Kondo effect [37, 38, 39], in which the localized magnetic moment (or spin) of the impurity ion is "quenched" by the spin polarization of the surrounding conduction electron density at temperatures below a characteristic temperature called the "Kondo temperature".

The Kondo effect is a temperature-dependent phenomenon which manifests itself in anomalies in the electrical resistivity, magnetic susceptibility, specific heat, thermoelectric power, etc. Kondo performed a perturbative expansion of the interaction Hamiltonian to order $\mathcal{J}^{3}$ and found that the magnetic impurity contribution $\rho_{m}$ to the electrical resistivity varied as $(-\ln T)$ for $T \gg T_{K}$ [39]; the addition of $\rho_{m}$ to the other electrical resistivity terms produced the anomalous minimum observed in low temperature measurements of the electrical resistivity of many alloys. These anomalies scale with the Kondo temperature $T_{K}$ :

$$
T_{K} \sim T_{F} \exp \left(-\frac{|\mathcal{J}|}{N\left(E_{F}\right)}\right),
$$

where $T_{F}$ is the Fermi temperature. The value of the Kondo temperature may span many orders of magnitude from $T_{K}$ $\sim 1 \mathrm{mK}$ to $T_{K} \sim 1000 \mathrm{~K}$. For the $\mathrm{La}_{1-x} \mathrm{Ce}_{x} \mathrm{Al}_{2}$ alloys, measurements of the normal state physical properties indicate that the Kondo temperature is in the range $T_{K} \sim 0.1$ to $0.6 \mathrm{~K}$ [40, 41, 42, 43, 44, 45]. Below $T_{K}$, the spins of the RE impurity ions are compensated by the spins of the surrounding conduction electrons, resulting in the formation of a "quasi-bound" many-body singlet ground state with a binding energy on the order of $k_{B} T_{K}$. This behavior is typical for $3 d$ transition metal impurities and the RE impurities $\mathrm{Ce}$ and $\mathrm{Yb}$.

Such a mechanism can also profoundly affect the superconducting properties. The most intriguing temperature regime is when $T_{c 0} \sim T_{K}$ for which Kondo scattering is most likely to affect superconductivity. Under these conditions, the singlet pairing of conduction electrons (with a binding energy $\sim k_{B} T_{c}$ ) competes with the many-body singlet pairing between conduction electrons and the RE impurity that form the "quasi-bound" state (with a binding energy $\sim k_{B} T_{K}$ ). This explains the anomalously large depression of $T_{c}$ due to the presence of the $\mathrm{Ce}$ impurities in the $\mathrm{La}_{1-x} \mathrm{Ce}_{x}$ and $\mathrm{La}_{1-x} \mathrm{Ce}_{x} \mathrm{Al}_{2}$ systems as displayed in Fig. 4. The superconducting critical temperatures for the $\mathrm{La}$ and $\mathrm{LaAl}_{2}$ hosts are $T_{c 0}=3.3 \mathrm{~K}$ and $6.0 \mathrm{~K}$, respectively, where $T_{K} \sim 0.5 \mathrm{~K}$ for each system. We will next discuss phenomena in the two regimes: $T_{c 0} \gg T_{K}$ and $T_{c 0} \ll T_{K}$.

In general, the CEF splitting of the ground state multiplet of a RE impurity must be taken into account when determining the impurity's effect on the superconducting properties. The exchange interaction between conduction electrons and the impurity spin depend on the CEF splitting of the levels at a given temperature and the depression of $T_{c}$ should reflect the CEF splitting of the Hund's rule ground state of the RE impurity ion [5]. The effect on $T_{c}$ may be summarized by two competing effects: (1) inelastic charge scattering of conduction electrons from the aspherical part of the $4 f$ shell, which has the effect of enhancing $T_{c}$, and (2) exchange scattering of conduction electrons which has the effect of depressing $T_{c}$. However, for the $\mathrm{La}_{1-x} \mathrm{Ce}_{x} \mathrm{Al}_{2}$ system, a number of experiments [46, 47, 48] have shown that the $\mathrm{Ce}^{3+} J=5 / 2$ Hund's rule multiplet is split by the CEF (produced by the fcc $\mathrm{C} 15$ crystal structure of the $\mathrm{LaAl}_{2}$ host) into a $\Gamma_{8}$ quartet and a ground state $\Gamma_{7}$ doublet with a splitting of the order of $100 \mathrm{~K}$. Since this splitting is large compared to $T_{c}$, the influence of the $\Gamma_{8}$ excited state quartet can be neglected at superconducting temperatures. Hence, the physical picture we have for the $\mathrm{La}_{1-x} \mathrm{Ce}_{x} \mathrm{Al}_{2}$ system in the $T_{c}$ $\gg T_{K}$ regime is characterized as one in which the conduction electrons must exchange-scatter from a ground state doublet with an effective spin of $1 / 2$ [6]. A full treatment of the CEF effects on RE impurity scattering in superconductors will not be given in this article, and we refer the interested reader to a number of comprehensive expositions on the subject [5, 19, 49].

Perhaps one of the more unusual and striking manifestations of the Kondo effect in the superconducting state is the phenomenon of reentrant superconductivity. In the regime where $T_{c 0} \gg T_{K}$, an alloy will first become superconducting upon cooling below an "upper" critical temperature $T_{c 1}$ and then remain in the superconducting state upon further cooling until it reaches a "lower" critical temperature $T_{c 2}$. At this point it "reenters" the normal state and remains normal for all temperatures $T \ll T_{c 2}$.

Reentrant superconductivity in the Kondo system $\mathrm{La}_{1-x} \mathrm{Ce}_{x} \mathrm{Al}_{2}$ can be seen in the $T_{c} / T_{c 0}$ vs. $x$ data displayed in Fig. 5 [50]. The solid curve is a guide to the eye and indicates that the $T_{c} / T_{c 0}$ vs. $x$ relationship is a single-valued function for the concentration range $x \leq 0.6$ at. $\%$. For this concentration range, the alloy remains superconducting for all temperatures $T<T_{c 1}$, i.e., the system does not "reenter" the normal state at lower temperature. For the concentration range $0.6 \leq x \leq 0.68$ at. $\%$, however, the $T_{c} / T_{c 0}$ vs. $x$ relationship is no longer a single-valued function of $x$; there now exists a set of lower critical temperatures $T_{c 2}$ that denote a return to the normal state. These "reentrant" points are indicated by the black squares and circles that appear along the solid curve below the symbol " $x$ " in Fig. 5. The symbol " $X$ " represents the estimated turning point which occurs at a $\mathrm{Ce}$ impurity concentration of $x \sim 0.67$ at. $\%$ in $\mathrm{La}_{1-x} \mathrm{Ce}_{x} \mathrm{Al}_{2}$ [5]. The upper 


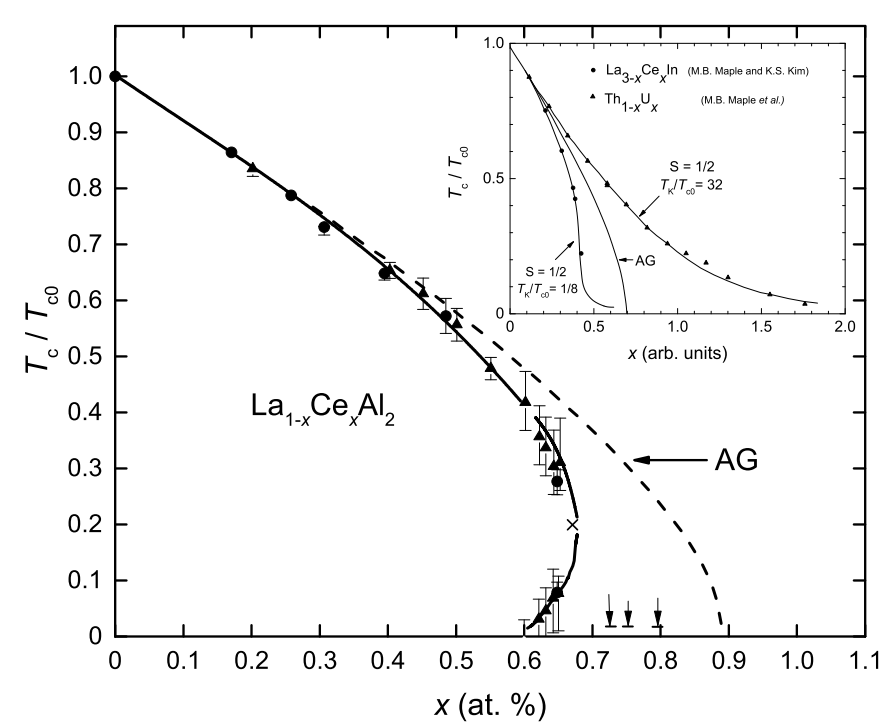

Figure 5: Reduced transition temperature $T_{c} / T_{c 0}$ versus impurity concentration $x$ for the $\mathrm{La}_{1-x} \mathrm{Ce}_{x} \mathrm{Al}_{2}$ system. The symbol $(\mathrm{x})$ indicates the estimated turning point of the $T_{c} / T_{c 0}$ vs. $x$ curve while the solid circles and triangles distinguish two separately prepared sets of alloys. The Abrikosov-Gor'kov (AG) curve (dashed) is shown for comparison. (from Ref. [50]) Inset: Two sets of experimental data in the $T_{c 0} \gg T_{K}$ and $T_{c 0} \ll T_{K}$ limits compared with AG and Müller-Hartmann-Zittarz (MHZ) theory. For $x=0$, the initial rates of depression $\left(d T_{c} / d x\right)_{x=0}$ for the three curves are equal. (from Ref. [51])

and lower superconducting transition temperatures, which occur above and below the turning point, respectively, were defined by superconducting transition curve midpoints (i.e., the $50 \%$ values compared to onset values) and the transition widths (represented by the vertical bars in the figure) were determined from the 10 and $90 \%$ values (relative to onset).

These unusual variations in the superconducting transition temperature in $\mathrm{La}_{1-x} \mathrm{Ce}_{x} \mathrm{Al}_{2}$ are attributed to the temperature dependence of the antiferromagnetic exchange scattering that occurs in these superconducting-Kondo systems. The destruction of superconductivity and the return to the normal state for temperatures $T \leq T_{c 2}$ is explained in terms of a pair-breaking theory based on a mechanism that depends on temperature $T$ and the impurity concentration $x$. The reader is referred to review articles by Müller-Hartmann [52] and Schlottmann [53, 54] for more information regarding the theory for superconducting-Kondo systems. We will provide only a brief treatment of this subject. For the case of $\mathcal{J}<0$, where $\alpha$ must be calculated to order beyond $\mathcal{J}^{2}$, the reduced pair-breaking parameter $\alpha / \alpha_{c r}$ was calculated in the theory of Müller-Hartmann and Zittartz (hereafter MHZ) and takes the form $[51,55]$ :

$$
\alpha(x, T)=x f(T) \sim x\left[\frac{\pi^{2} S(S+1)}{\left[\ln \left(T / T_{K}\right)\right]^{2}+\pi^{2} S(S+1)}\right] .
$$

It is easy to see from this relation that the temperaturedependent pair-breaking parameter $\alpha(T, x)$ is maximum at $T_{K}$, and it is indeed the case that the suppression rate of $T_{c}$ is largest when $T_{c 0} \sim T_{K}$ and smaller in the regimes $T_{c 0} \gg T_{K}$ and $T_{c 0} \ll T_{K}$ [56]. According to MHZ theory, the supercon- ducting properties may be characterized by $\alpha(T, x)$ such that the universal relation $T_{c} / T_{c 0}$ vs. $\alpha / \alpha_{c r}$ based on AG theory (Equation 5) still applies; however, in order to determine the functional dependence of $T_{c}(x)$, the temperature-dependent reduced pair-breaking parameter $\alpha(T, x) / \alpha_{c r}$ cannot simply be replaced by $x / x_{c r}$ in Equation 5. In order to determine the $x$ dependence for $T_{c}$ in the regime $T_{c 0} \gg T_{K}$, the universal relation for $T_{c} / T_{c 0}$ as a function of $\alpha / \alpha_{c r}$ in Equation 5 must be solved simultaneously with Equation 11. The calculation based on MHZ theory is sensitive to the relative magnitude of $T_{c 0}$ compared to that of the Kondo temperature $T_{K}$. In the regime where $T_{c 0} \lesssim 8 T_{K}$ (and for an impurity spin of $S=1 / 2$ ), the MHZ theory predicts that $T_{c}$ is a single-valued, monotonically-decreasing function of $x$, and approaches the exponential form $T_{c} \sim e^{-[\beta+\gamma x]^{1 / 2}}$ in the $T_{c 0}$ $\ll T_{K}$ limit [6]. This $x$ dependence of $T_{c}$ describes the $T_{c}$ vs. $x$ data for the alloy $\mathrm{Th}_{1-x} \mathrm{U}_{x}$ [57] shown in the inset of Fig. 5.

In the regime where $T_{c 0} \gtrsim 8 T_{K}$, the MHZ theory predicts the existence of more than one solution for $T_{c}(x)$ within a suitable concentration range. As the temperature is lowered, an alloy containing the requisite amount of RE impurities becomes superconducting at $T_{c 1}$, remains superconducting to $T_{c 2}$, below which it reenters the normal state; remarkably, the MHZ theory predicts that upon further cooling, it should become superconducting again at $T_{c 3}$. The first two critical temperatures $T_{c 1}(x)$ and $T_{c 2}(x)$ have been verified by experiments on the $\mathrm{La}_{1-x} \mathrm{Ce}_{x} \mathrm{Al}_{2}$ system (see Fig. 5) [50, 58]. The existence of the third transition at $T_{c 3}(x)$, however, has not yet been established experimentally. Selected samples of $\mathrm{La}_{1-x} \mathrm{Ce}_{x} \mathrm{Al}_{2}$ were measured down to $6 \mathrm{mK}\left(T_{c} / T_{c 0} \sim 2 \times 10^{-3}\right)$ but they did not exhibit any evidence for a third transition back to the superconducting state as predicted by MHZ theory [50]. Either $T_{c 3}(x)$ is less than $6 \mathrm{mK}$ or it simply does not exist. A theory for superconductingKondo systems proposed by Schlottmann predicts that in the $T_{c 0} \gg T_{K}$ regime a system returns to the normal state at a second critical temperature $T_{c 2}(x)$ below $T_{c 1}(x)$ but that there is no transition back to the superconducting state at $T_{c 3}(x)$ [53]. We will revisit reentrant superconductivity in our discussion of ferromagnetic superconductors but note that the mechanism in these cases is distinct from that in Kondo superconductors.

In the late 1960s, investigations of the pressure dependence of $T_{c}$ were carried out on three superconductors containing Ce impurities, $\mathrm{La}_{3} \mathrm{In}$ [60], $\mathrm{La}$ [61], and $\mathrm{LaAl}_{2}$ [62]. Measurements on the systems $\mathrm{La}_{3-x} \mathrm{Ce}_{x} \mathrm{In}$ and $\mathrm{La}_{1-x} \mathrm{Ce}_{x}$ under pressure revealed that the $\mathrm{Ce}$ impurities, which carried well defined magnetic moments and generated a Kondo effect at atmospheric pressure, appeared to undergo a continuous demagnetization under pressure. This was reflected in the behavior of the pressure dependence of the rate of depression of $T_{c}$ by the Ce additions that initially increased, passed through a maximum, and then decreased. In later work, it was observed that a similar demagnetization of the Ce impurities occurs in the $\mathrm{La}_{1-x} \mathrm{Ce}_{x}$ system when Th is substituted for La [59]. With increasing Th concentration $y$ in the $\left(\mathrm{La}_{1-y} \mathrm{Th}_{y}\right)_{1-x} \mathrm{Ce}_{x}$ system, the rate of the initial depression of $T_{c}$ with Ce concentration $x$ passes through a pronounced maximum and $T_{c}$ vs. $x$ changes continuously from curves with negative curvature and reentrant behavior to curves with positive curvature and exponential-like shapes [59]. This 


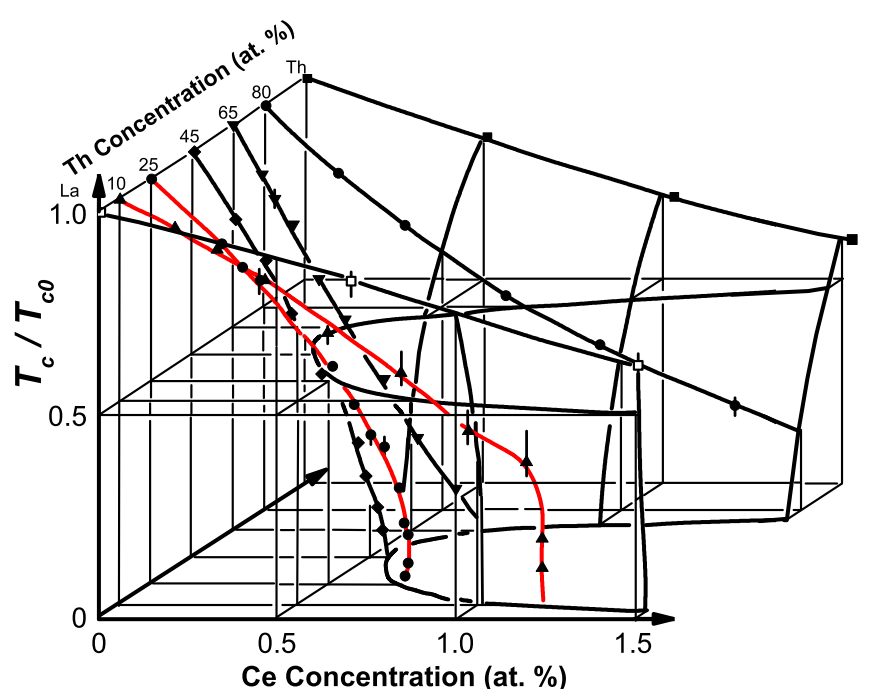

Figure 6: Normalized transition temperature $T_{c} / T_{c 0}$ vs. Ce concentration $x$ and Th concentration $y$ for $\left(\mathrm{La}_{1-y} \mathrm{Th}_{y}\right)_{1-x} \mathrm{Ce}_{x}$. The curves of $T_{c} / T_{c 0}$ vs. Ce concentration (shown in red) for the $\left(\mathrm{La}_{0.90} \mathrm{Th}_{0.10}\right)_{1-x} \mathrm{Ce}_{x}$ and the $\left(\mathrm{La}_{0.75} \mathrm{Th}_{0.25}\right)_{1-x} \mathrm{Ce}_{x}$ systems are reentrant. (from Ref. [59])

is illustrated in the three dimensional plot of the $T_{c}$ vs. Ce concentration curves for various Th concentrations in Fig. 6. Concomitantly, the normal-state magnetic susceptibility [63] and electrical resistivity [64] evolve continuously from magnetic to nonmagnetic behavior. A self-consistent analysis of the rates of the initial depression of $T_{c}$ with $x$ and the value of the specific heat jump at $T_{c}$ showed that the MHZ theory provides a good description of the data from $T_{K} / T_{c 0} \sim 10^{-1}$ to $10^{2}[65,66]$.

Experiments on several superconducting matrix-impurity systems in which the impurity ion exhibited nonmagnetic behavior at superconducting temperatures revealed that the $T_{c}$ vs. $x$ curves have positive curvature and could be described well by a modified exponential relation of the form:

$$
T_{c} / T_{c o}=A /(1-D x)
$$

This expression was proposed by Kaiser [67] who considered the effect of nonmagnetic resonant $d$ - and $f$-electron states of impurity ions on superconductivity. In Equation 12, $A$ and $D$ are fitting parameters that can be related to quantities in the FriedelAnderson model. The systems investigated included $\mathrm{Th}_{1-x} \mathrm{U}_{x}$ [6], $\mathrm{Th}_{1-x} \mathrm{Ce}_{x}$ [68], and $\mathrm{Al}_{1-x} \mathrm{Mn}_{x}$ [69]. The nonmagnetic limit corresponds to $T_{o} / T_{c 0} \gg 1$, where $T_{o}$ is a characteristic temperature that separates low temperature nonmagnetic behavior from high temperature magnetic behavior. However, in this limit it is not clear that a description based on the exchange interaction is appropriate, since the system is in the short-lived local moment regime and it may be more appropriate to identify $T_{o}$ with a spin fluctuation temperature, rather than the Kondo temperature $T_{K}$. For more information regarding the high $T_{K}$ regime, the reader can consult Ref. [6].

\section{Ternary Compounds: Magnetically Ordered Supercon- ductors}

The investigations of binary and pseudobinary superconducting compounds containing magnetic impurity ions revealed that $T_{c}$ is driven to $0 \mathrm{~K}$ at small impurity concentrations of only a few atomic percent. The existence of the very strong pair-breaking mechanism associated with the exchange interaction made it challenging to find materials in which to study the interplay of superconductivity and long-range magnetic order. An obvious solution for avoiding the destructive effects of the exchange interaction was to search for systems in which the exchange interaction was particularly weak. Matthias and coworkers were the first to exploit the weak exchange interactions in the pseudobinary compounds $\mathrm{Ce}_{1-x} \mathrm{RE}_{x} \mathrm{Ru}_{2}$ and $\mathrm{Y}_{1-x} \mathrm{RE}_{x} \mathrm{Os}_{2}[3,70]$. The depression of $T_{c}$ was less rapid in these systems and as a result the $T_{c}(x)$ curves intersect the $T_{M}(x)$ curves creating a region of overlap in which it was thought that superconductivity coexisted with long-range magnetic order [71, 72]. However, the magnetic ordering was best described as short-range "glassy" magnetic order.

This changed completely in the 1970s with the discovery of two new classes of ternary compounds that were the first to exhibit long-range magnetic order in the superconducting state: (1) the ternary RE molybdenum chalcogenides $\mathrm{REMo}_{6} \mathrm{X}_{8}$ $(X=S, S e)$ and (2) the RE rhodium borides $R_{E R h} B_{4}$. In 1980, a third class of ternary magnetic superconductors was discovered, the $\mathrm{RE}$ rhodium stannides $\mathrm{RERh}_{1.1} \mathrm{Sn}_{3.6}$ and in the 1990s, another class of magnetic superconductors was discovered, the quaternary borocarbide compounds $\mathrm{RENi}_{2} \mathrm{~B}_{2} \mathrm{C}$, which provided another platform to study the interplay between superconductivity and long-range magnetic order [73]. We will now provide a selective review of results from studies on compounds from these classes of magnetic superconductors.

\section{1. $R E R h_{4} B_{4}, R_{E M o} X_{8}(X=S, S e)$, and $E r R h_{1.1} S n_{3.6}$}

The discovery of superconductivity in the ternary molybdenum chalcogenides (or Chevrel phases) $\mathrm{MMo}_{6} \mathrm{~S}_{8}(\mathrm{M}=$ $\mathrm{Cu}, \mathrm{Zn}, \mathrm{Mg}, \mathrm{Ag}, \mathrm{Cd}, \mathrm{Sn}, \mathrm{Pb}$, and RE) in 1972 [7, 8], the RE rhodium boride ternary compounds $\mathrm{RERh}_{4} \mathrm{~B}_{4}$ in 1977 [9], and the rare earth rhodium stannides $\mathrm{RERh}_{1.1} \mathrm{Sn}_{3.6}$ [74] in 1980 , resulted in a burst of experimental and theoretical investigation of $\mathrm{REMo}_{6} \mathrm{X}_{8}(\mathrm{X}=\mathrm{S}, \mathrm{Se})[75,76,77,78,79,80,81,82,83,84,85]$ and $\mathrm{RERh}_{4} \mathrm{~B}_{4}[83,84,86,87,88]$ compounds, which provided a chance to explore a host of new and interesting phenomena including: (1) the coexistence of long-range antiferromagnetic order and superconductivity, (2) the competition between ferromagnetic order and superconductivity, which can lead to reentrant superconductivity, (3) the emergence of a longwavelength oscillatory magnetic state that coexists with the superconducting state within a narrow temperature range, below which, ferromagnetic order destroys superconductivity, and (4) magnetic field induced superconductivity (MFIS), whereby an applied magnetic field can compensate the exchange field produced by the RE magnetic ions, enabling superconductivity to emerge.

The Curie (ferromagnetic ordering) temperature $T_{M}$, the 


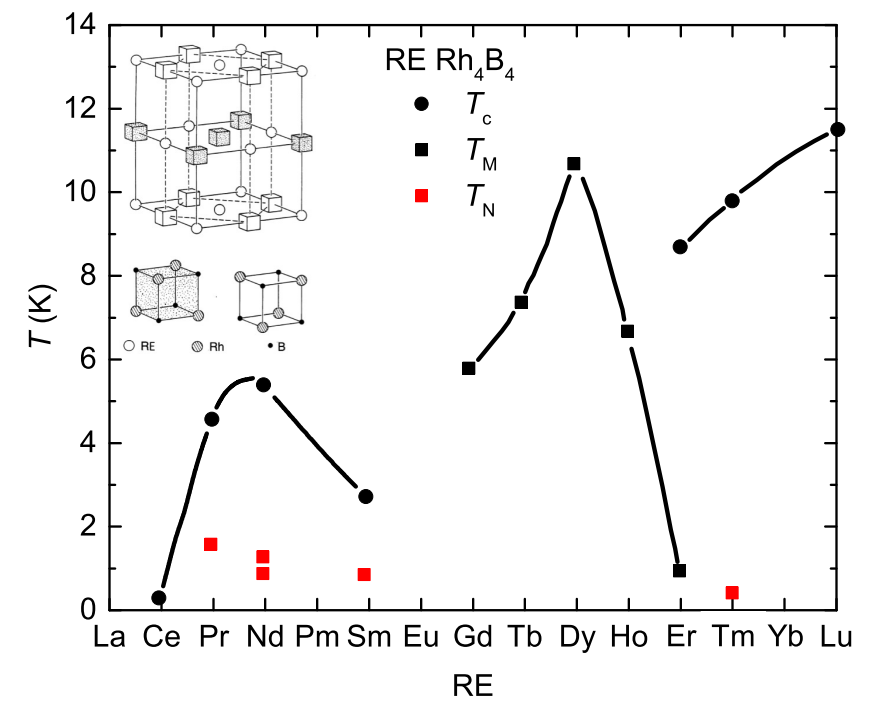

Figure 7: Superconducting transition temperature $T_{c}$, ferromagnetic ordering temperature (Curie temperature) $T_{M}$, and antiferromagnetic ordering temperature (Néel temperature) $T_{N}$ vs. rare earth (RE) impurity for the $\mathrm{RERh}_{4} \mathrm{~B}_{4}$ compounds. (from Refs. [9, 86, 89, 87, 90, 88, 91, 92]) Inset: Crystal structure of $\mathrm{RERh}_{4} \mathrm{~B}_{4}$ compounds with the primitive tetragonal unit cell represented by the dashed outline. For clarity, the $\mathrm{Rh}_{4} \mathrm{~B}_{4}$ clusters are not drawn to scale. (from Ref. [93])

Néel (antiferromagnetic ordering) temperature $T_{N}$, and $T_{c}$ are plotted versus RE element in Fig. 7 for the compounds $\mathrm{RERh}_{4} \mathrm{~B}_{4}$ (except for the compounds $\mathrm{PmRh}_{4} \mathrm{~B}_{4}$ and $\mathrm{EuRh}_{4} \mathrm{~B}_{4}$ that have not been synthesized). It is evident that the compounds containing $\mathrm{RE}$ ions with partially-filled $4 f$-electron shells all undergo some type of magnetic ordering below $T_{c}$ at temperatures $T_{M}$ or $T_{N} \sim 1 \mathrm{~K}$. In particular, antiferromagnetic order is observed for the superconducting compounds $\mathrm{RERh}_{4} \mathrm{~B}_{4}(\mathrm{RE}=\mathrm{Ce}$ [94], Pr [94], Nd [87, 90], Sm [88], Tm [91, 92]) while ferromagnetic order is observed for the superconducting compound $\mathrm{ErRh}_{4} \mathrm{~B}_{4}[86,89]$.

\subsubsection{Superconducting $R h_{4} B_{4}$ and $M_{6} o_{8}(X=S$, Se) Clusters}

As the name implies, a ternary compound consists of three different elements, each of which occupies one or more crystallographic sites throughout the lattice. Although a pseudobinary compound also consists of three different elements, only two elements occupy specific crystallographic sites within the lattice; the third element substitutes for one (or both) of the other two elements. The existence of distinct crystallographic sites for the three different elements is crucial for some of the phenomena that are observed in these ternary compounds.

The crystal structure of the $\mathrm{REMo}_{6} \mathrm{X}_{8}(\mathrm{X}=\mathrm{S}, \mathrm{Se})$ system (related to the $\mathrm{Mo}_{6} \mathrm{X}_{8}$ binary structures) is rhombohedral with space group $R \overline{3}$ [7, 95, 96], while the crystal structure of the $\mathrm{RERh}_{4} \mathrm{~B}_{4}$ system is isostructural to the $\mathrm{CeCo}_{4} \mathrm{~B}_{4}$ tetragonal structure with space group $P 4_{2} / n m c$ [97]. The two different crystal structures, however, are similar in that: (1) they exhibit a lattice of molecular "clusters" that supply the material with superconducting electrons, (2) they contain an ordered RE sublattice in which the RE ions develop long-range magnetic or- der via the Ruderman-Kittel-Kasuya-Yosida (hereafter RKKY) mechanism [98, 99, 100], and (3) the distance between the RE ions and the molecular "clusters" is relatively large, leading to a reduced exchange interaction and the preservation of superconducting electron pairs, which allows for the coexistence of superconductivity and long-range magnetic order.

The tetragonal crystal structure of the $\mathrm{RERh}_{4} \mathrm{~B}_{4}$ compounds is displayed as an inset in Fig. 7 in a manner that emphasizes the superconducting $\mathrm{Rh}_{4} \mathrm{~B}_{4}$ molecular "clusters" $[93,102]$. For a detailed discussion and illustration of the more complicated crystal structure and clustering observed for the $\mathrm{REMo}_{6} \mathrm{X}_{8}$ compounds, the reader is referred to the review article by Chevrel and Sergent in Ref. [96] and an overview of their superconducting properties may be found in another article in this special issue by Peña. The source of the observed superconductivity in the $\mathrm{REMo}_{6} \mathrm{X}_{8}(\mathrm{X}=\mathrm{S}, \mathrm{Se})$ and $\mathrm{RERh}_{4} \mathrm{~B}_{4}$ compounds is attributed to the $4 d$ electrons from Mo and $\mathrm{Rh}$; these $4 d$ electrons are somewhat confined to the $\mathrm{Mo}_{6} \mathrm{X}_{8}(\mathrm{X}=$ $\mathrm{S}, \mathrm{Se}$ ) and $\mathrm{Rh}_{4} \mathrm{~B}_{4}$ molecular "clusters" and, therefore, tend to be isolated from the pair-breaking effects associated with the lattice of magnetic RE ions. In the case of the $\mathrm{RERh}_{4} \mathrm{~B}_{4}$ compounds, the relative confinement of the $\mathrm{Rh} 4 d$ electrons within the $\mathrm{Rh}_{4} \mathrm{~B}_{4}$ molecular "clusters" are reflected in both the low $4 d$ electron density observed at the RE sites and also in the resonances in the electronic density of states at the Fermi level $[103,104,105]$.

\subsubsection{Ferromagnetic Superconductors and Reentrant Super- conductivity}

In Section 2.2, we found that certain superconductors containing paramagnetic impurities with single-ion Kondo temperatures $T_{K}$ in the $T_{c 0} \gg T_{K}$ regime could exhibit reentrant superconductivity. Reentrant superconductivity was later observed in 1977 in the superconducting ternary compounds $\mathrm{ErRh}_{4} \mathrm{~B}_{4}$ and $\mathrm{Ho}_{1.2} \mathrm{Mo}_{6} \mathrm{~S}_{8}$ at the University of California, San Diego [86] and the University of Geneva [106], respectively. Shortly thereafter, in 1980, reentrant superconductivity was also observed in

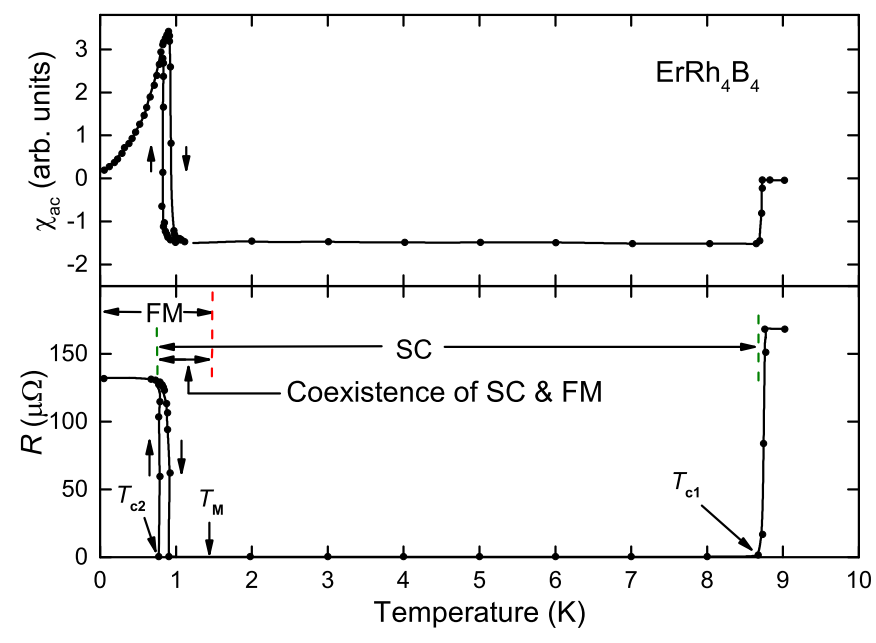

Figure 8: AC magnetic susceptibility $\chi_{A C}$ and AC electrical resistance vs. temperature data for $\mathrm{ErRh}_{4} \mathrm{~B}_{4}$ in zero applied magnetic field. (from Ref. [101]) 


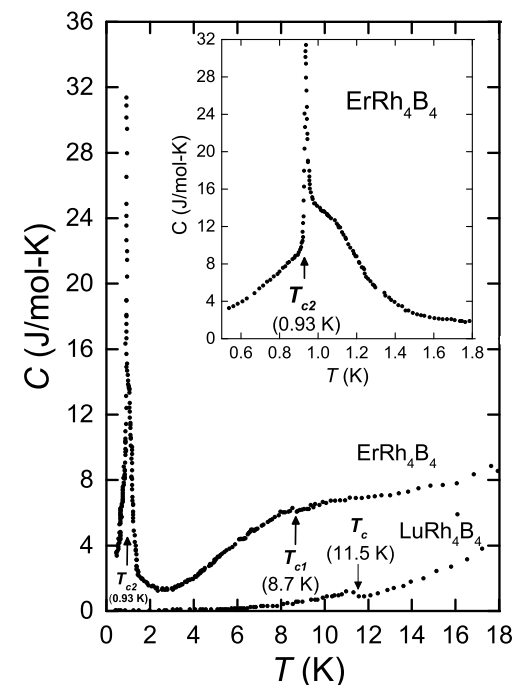

Figure 9: Specific heat vs. temperature (C vs. T) for $\mathrm{ErRh}_{4} \mathrm{~B}_{4}$ and $\mathrm{LuRh}_{4} \mathrm{~B}_{4}$ The inset shows the plot of $\mathrm{C}$ vs. $T$ for $\mathrm{ErRh}_{4} \mathrm{~B}_{4}$ near the reentrant superconducting transition at $T_{c 2}$. (from Ref. [23]; based on data from Ref. [93])

the rare earth rhodium stannide $\mathrm{ErRh}_{1.1} \mathrm{Sn}_{3.6}$ [74]. The destruction of superconductivity at a second critical temperature $T_{c 2}$ in these ternary superconductors is due to the onset of long-range ferromagnetic order at a temperature $T_{M} \sim T_{c 2}$ rather than the temperature-dependent pair-breaking mechanism related to the Kondo effect. It is clear from Fig. 7 that, for the superconducting $\mathrm{RERh}_{4} \mathrm{~B}_{4}$ compounds (Ce excluded), the $T_{c}$ values are greater than $\sim 3 \mathrm{~K}$; these are significantly higher than the magnetic ordering temperatures which are on the order of $\sim 1 \mathrm{~K}$. $\mathrm{ErRh}_{4} \mathrm{~B}_{4}$ is the only superconductor $\left(T_{c} \sim 8.5 \mathrm{~K}\right)$ that exhibits ferromagnetic order with $T_{M} \sim 1 \mathrm{~K}$. This value of $T_{M}$ corresponds roughly to the temperature at which the superconductor returns to the normal state, $T_{c 2} \sim 1 \mathrm{~K}$. The correspondence between $T_{c 2}$ and $T_{M}$ can also be seen in the measurements of electrical resistance $R$ vs. $T$ and AC magnetic susceptibility $\chi_{A C}$ vs. $T$ as displayed in Fig. 8. It is clear from the figure that $\mathrm{ErRh}_{4} \mathrm{~B}_{4}$ first becomes superconducting at $T_{c 1} \sim 8.5 \mathrm{~K}$ and then exhibits ferromagnetic order at the Curie temperature $T_{M} \sim 1.2 \mathrm{~K}$ before returning to a ferromagnetic normal state at a lower critical temperature $T_{c 2} \sim 0.9 \mathrm{~K}$ such that $T_{c 2}<T_{M}<T_{c 1}$. The observed thermal hysteresis at $T_{c 2}$ in the measurements of $R$ vs. $T$ and $\chi_{A C}$ vs. $T$ as shown in Fig. 8 as well as features in measurements of the specific heat in the vicinity of $T_{c 2}$ (see Fig. 9) reflect the first-order character of the phase transition [93, 107].

Similar measurements of DC electrical resistance $R$ vs. $T$ were performed for the series of compounds $\mathrm{RE}_{1.2} \mathrm{Mo}_{6} \mathrm{~S}_{8}$ (RE $=\mathrm{Tb}, \mathrm{Dy}, \mathrm{Ho}, \mathrm{Er}, \mathrm{Tm})$ in applied magnetic fields $H[106,77]$. It was found that only the $\mathrm{Ho}_{1.2} \mathrm{Mo}_{6} \mathrm{~S}_{8}$ compound exhibited reentrant superconductivity in which the normal state is restored for $H=0$ as displayed in Fig. 10. As with the rare earth rhodium boride compounds $\mathrm{RERh}_{4} \mathrm{~B}_{4}$, many of the superconducting rare earth molybdenum chalcogenide compounds in the series $\mathrm{REMo}_{6} \mathrm{~S}_{8}$ (with the exception of $\mathrm{RE}=\mathrm{Ce}, \mathrm{Eu}$, and $\mathrm{Er}$ ) also exhibit superconductivity with $T_{c}>1.1 \mathrm{~K}$ that are consistently higher than their respective magnetic ordering tempera- tures (usually less than $1 \mathrm{~K}$ ) [77]. We will discuss examples of pseudoternary magnetic superconductors in Section 3.2 and the $\mathrm{RE}$ series of quaternary compounds $\mathrm{RENi}_{2} \mathrm{~B}_{2} \mathrm{C}$ in Section 4 in which the magnetic (antiferromagnetic) ordering temperature $T_{N}$ is greater than $T_{c}$.

$\mathrm{Ho}_{1.2} \mathrm{Mo}_{6} \mathrm{~S}_{8}, \mathrm{ErRh}_{4} \mathrm{~B}_{4}$, and $\mathrm{ErRh}_{1.1} \mathrm{Sn}_{3.6}$ are the only superconducting ternary compounds that exhibit long-range ferromagnetic order below $T_{c}$; ferromagnetic order destroys superconductivity in each compound [86, 106, 74]. Neutrondiffraction experiments on polycrystalline samples of $\mathrm{ErRh}_{4} \mathrm{~B}_{4}$ [89] and $\mathrm{Ho}_{1.2} \mathrm{Mo}_{6} \mathrm{~S}_{8}$ [108] indicate that each compound exhibits a ferromagnetic and normal ground state. The survival of the ferromagnetic state at the expense of superconductivity follows from a larger decrease in the thermodynamic free energy for the ferromagnetic state relative to the paramagnetic normal state [109] when compared with the decrease in free energy observed in the superconducting state relative to the paramagnetic normal state. The decrease in the thermodynamic free energy of the ferromagnetic state is roughly $\sim c N k_{B} T_{M}$ where $c$ is the atomic fraction of magnetic ions in the sample and $N$ is the total number of atoms in the sample. In comparison, the decrease in free energy of the superconducting state is $~$ $\left(k_{B} T_{c} / E_{F}\right) N k_{B} T_{c}$ where the factor $\left(k_{B} T_{c} / E_{F}\right)$ is the proportion of conduction electrons that participate in superconductivity. Typically, $\mathrm{c} \gg\left(k_{B} T_{c} / E_{F}\right)$ so that the ferromagnetic state will be favored as the ground state. Nevertheless, there appears to be a narrow temperature range $T_{c 2}<T<T_{M}$ within which superconductivity and ferromagnetism coexist. In this temperature region, small-angle neutron-diffraction experiments have confirmed the existence of an oscillatory magnetic state with a wavelength on the order of $\sim 100 \AA$ in both $\mathrm{Ho}_{1.2} \mathrm{Mo}_{6} \mathrm{~S}_{8}$ and $\mathrm{ErRh}_{4} \mathrm{~B}_{4}[110,111,112]$. Neutron-diffraction measurements on $\mathrm{ErRh}_{4} \mathrm{~B}_{4}$ indicate that superconductivity and ferromagnetic order coexist macroscopically, while superconductivity and the sinusoidally modulated magnetic state coexist microscopically between $T_{c 2}$ and $T_{c 1}$ [110].

Reentrant superconductivity due to the onset of ferromagnetic order was first predicted in 1964 by Gor'kov and Rusinov [32]. Their theory attributes the destruction of superconductivity to the magnetic pair-breaking that results from the non-zero average polarization of the conduction electron spins that arises from the ferromagnetic alignment of the magnetic moments of the RE ions [109]. It was believed that the net spin polarization of the conduction electrons could be reduced by the spin-orbit and exchange interactions and, hence, preserve the superconducting state and its coexistence with a ferromagnetically ordered lattice of RE ions.

\subsubsection{Coexistence of Antiferromagnetic Order and Supercon- ductivity}

In the previous section, we discussed the compounds $\mathrm{Ho}_{1.2} \mathrm{Mo}_{6} \mathrm{~S}_{8}$ and $\mathrm{ErRh}_{4} \mathrm{~B}_{4}$, that exhibit a suppression of superconductivity by the onset of ferromagnetic order. In contrast, the coexistence between antiferromagnetic order and superconductivity is more commonly observed. Such coexistence was first discovered in the molybdenum chalcogenides $\mathrm{REMo}_{6} \mathrm{~S}_{8}$ $(\mathrm{RE}=\mathrm{Tb}, \mathrm{Dy}, \mathrm{Er})$ [77] and $\mathrm{ErMo}_{6} \mathrm{Se}_{8}$ [81] and subsequently 


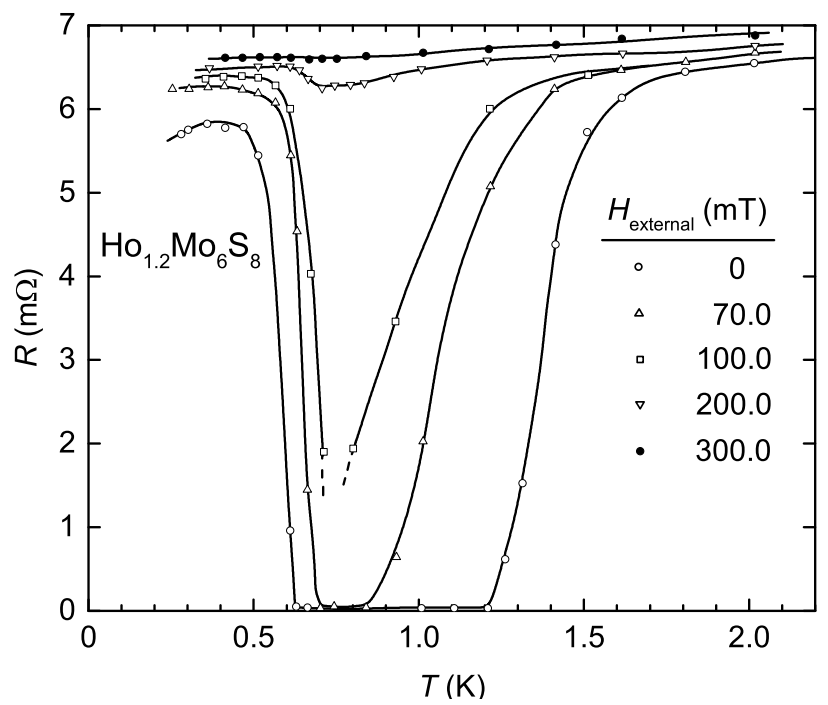

Figure 10: DC electrical resistance vs. temperature data for $\mathrm{Ho}_{1.2} \mathrm{Mo}_{6} \mathrm{~S}_{8}$ in static external magnetic fields of $H_{\text {external }}=0,70,100,200$, and $300 \mathrm{mT}$. (from Ref. [106])

in other ternary compounds $\mathrm{REMo}_{6} \mathrm{X}_{8}(\mathrm{X}=\mathrm{S}, \mathrm{Se})[79,85]$ and $\mathrm{RERh}_{4} \mathrm{~B}_{4}(\mathrm{RE}=\mathrm{Nd}, \mathrm{Sm}, \mathrm{Tm})$ [84]. More than a decade earlier in 1963, Baltensperger and Strässler predicted on theoretical grounds that these two ordered states could coexist [113]. Qualitatively, the compatibility of superconductivity and antiferromagnetic order follows from the fact that the average of the spin polarization of the magnetic ions in the antiferromagnetic state is zero when taken over a length scale on the order of the superconducting coherence length $\xi_{0}$. The absence of total spin polarization within an antiferromagnetic state reduces the potential strength of magnetic pair-breaking that might otherwise destroy superconductivity [114]. Nevertheless, the superconducting phase is modified significantly as the system begins to order antiferromagnetically. The effects of the magnetic order are reflected in strong anomalies in various superconducting properties near $T_{N}$ as illustrated in several systems discussed below. Particular attention will be given to the anomalies observed in the upper critical field $H_{c 2}$ vs. $T$ data.

The upper critical field curves $H_{c 2}(T)$, determined from electrical resistivity measurements in applied magnetic fields, for the antiferromagnetic superconductors $\mathrm{RERh}_{4} \mathrm{~B}_{4}(\mathrm{RE}=\mathrm{Nd}$, $\mathrm{Sm})[87,88]$, the ferromagnetic superconductor $\mathrm{ErRh}_{4} \mathrm{~B}_{4}$ [86], and the non-magnetic superconductor $\mathrm{LuRh}_{4} \mathrm{~B}_{4}$ [84] are shown in Fig. 11. The measurements are on polycrystalline samples which do not provide any information about the anisotropy of the upper critical field. Regarding the antiferromagnetic compounds, $\mathrm{NdRh}_{4} \mathrm{~B}_{4}$ and $\mathrm{SmRh}_{4} \mathrm{~B}_{4}$, the salient features that occur at their respective $T_{N}$ values, as shown in Fig. 11 (b) and Fig. 11 (c), respectively, suggest that the suppression of superconductivity by the magnetic field is affected by the onset of the antiferromagnetic order. Strikingly similar features in the $H_{c 2}(T)$ curves are shown in the inset of Fig. 12 for the various antiferromagnetic superconducting compounds $\mathrm{RE}_{1.2} \mathrm{Mo}_{6} \mathrm{~S}_{8}(\mathrm{RE}=$ $\mathrm{Gd}, \mathrm{Tb}, \mathrm{Dy})$ [77, 79]. The anomalous features in these $H_{c 2}(T)$ curves for $\mathrm{RE}_{1.2} \mathrm{Mo}_{6} \mathrm{~S}_{8}(\mathrm{RE}=\mathrm{Gd}, \mathrm{Tb}, \mathrm{Dy})$ occur at tempera-
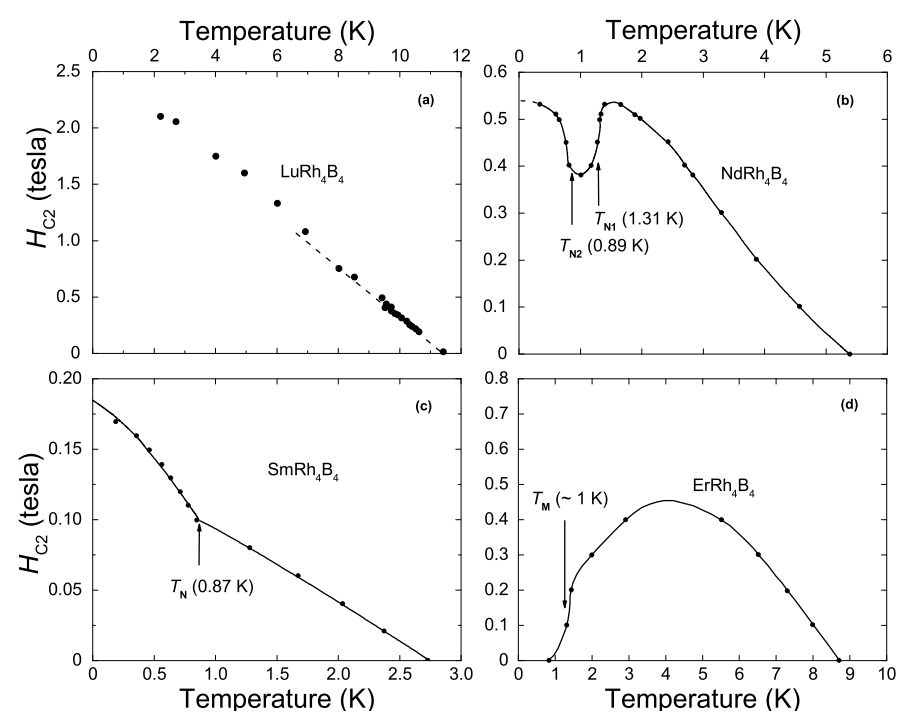

Figure 11: Upper critical fields $H_{c 2}$ for $\mathrm{LuRh}_{4} \mathrm{~B}_{4}$ [115], $\mathrm{NdRh}_{4} \mathrm{~B}_{4}$ [87], $\mathrm{SmRh}_{4} \mathrm{~B}_{4}$ [88], and $\mathrm{ErRh}_{4} \mathrm{~B}_{4}[116,93]$.

tures that correspond to the antiferromagnetic ordering of the RE magnetic moments as observed in the plots of the sublattice magnetization vs. temperature for $\mathrm{RE}_{1.2} \mathrm{Mo}_{6} \mathrm{~S}_{8}(\mathrm{RE}=\mathrm{Gd}, \mathrm{Tb}$, Dy) (see the main panel of Fig. 12).

For the compound $\mathrm{NdRh}_{4} \mathrm{~B}_{4}\left(T_{c} \sim 5.3 \mathrm{~K}\right)$, there is a significant and abrupt downturn in $H_{c 2}(T)$ at $T_{N 1} \sim 1.31 \mathrm{~K}$ followed by a rapid increase at a lower temperature $T_{N 2} \sim 0.89 \mathrm{~K}$. The rapid decrease at $T_{N 1}$ and subsequent increase at $T_{N 2}$ in the upper critical field reflect the existence of two antiferromagnetic phase transitions in $\mathrm{NdRh}_{4} \mathrm{~B}_{4}$. Two distinct antiferromagnetic structures were confirmed in neutron-scattering measurements [90], which showed that the Nd magnetic moments order antiferromagnetically along the $c$ axis (see inset in Fig. 7 for an illustration of the $\mathrm{NdRh}_{4} \mathrm{~B}_{4}$ crystal structure). The lower temperature structure $\left(T<T_{N 2}\right)$ is characterized by a sinusoidal variation in the magnetism along the crystallographic [110] direction with a wavelength $\lambda \sim 45.2 \AA$ and the higher temperature structure $\left(T_{N 2}<T<T_{N 1}\right)$ obeys a sinusoidal variation along the crystallographic [100] direction with a wavelength $\lambda \sim 46.5 \AA$. Qualitatively, the decrease in $H_{c 2}(T)$ at $T_{N 2}=1.31 \mathrm{~K}$ indicates that the antiferromagnetic order associated with the higher temperature structure causes additional pair-breaking, thus requiring a smaller external magnetic field to destroy superconductivity; conversely, the increase in the upper critical field curve near $T_{N 2}$ suggests that this type of magnetic order is more compatible with superconductivity [24]. Significant theoretical effort, rooted in the multiple pair-breaking theory that evolved throughout the 1960s and 1970s [117], has been made in order to explain the downturns in the $H_{c 2}(T)$ curves that occur just below $T_{N}$ as observed in $\mathrm{NdRh}_{4} \mathrm{~B}_{4}$ as well as in the antiferromagnetic superconductors from the $\mathrm{REMo}_{6} \mathrm{~S}_{8}$ series [114].

The superconducting compound $\mathrm{SmRh}_{4} \mathrm{~B}_{4}\left(T_{c} \sim 2.72 \mathrm{~K}\right)$ also exhibits a feature in its $H_{c 2}(T)$ data. At the Néel temperature $T_{N} \sim 0.87 \mathrm{~K}$, there is a noticeable cusp and sudden increase in the slope of $H_{c 2}(T)$ as temperature is decreased. Qualitatively, this upturn can be explained in terms of a reduction in 


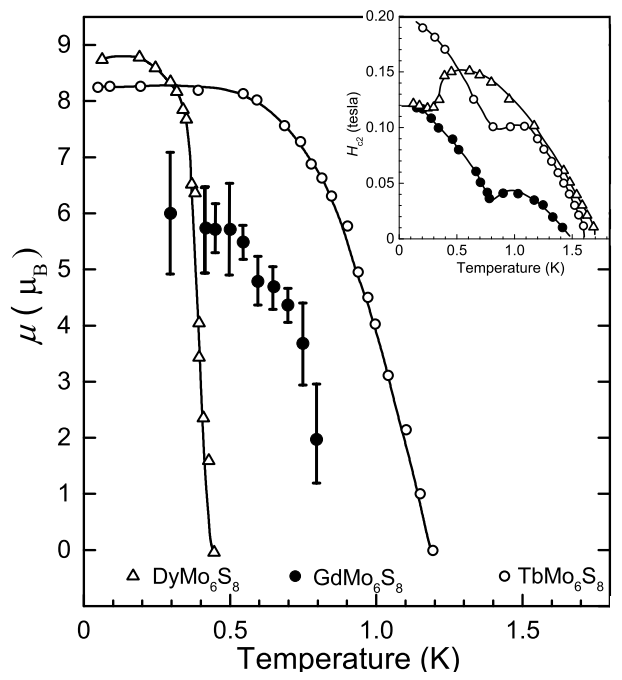

Figure 12: A plot of the magnetic moment $\mu$ vs. temperature $T$ for the compounds $\mathrm{REMo}_{6} \mathrm{~S}_{8}$ with $\mathrm{RE}=\mathrm{Gd}, \mathrm{Tb}$, and Dy as determined from neutrondiffraction experiments. The inset shows upper critical field curves $H_{c 2}(T)$ for $\mathrm{REMo}_{6} \mathrm{~S}_{8}(\mathrm{RE}=\mathrm{Gd}, \mathrm{Tb}$, and Dy). (from Ref. [23]; based on data from Ref. [79])

magnetic pair-breaking that is associated with the onset of antiferromagnetic order. Such behavior is accounted for in a model of antiferromagnetic superconductors in which antiferromagnetic order at $T_{N}$ quenches the pair-breaking effects due to the local $\mathrm{Sm}^{3+}$ magnetic moments and leads to an overall reduction in the net magnetization of the sample [118, 119]. A lambdatype anomaly was observed at $T_{N}=0.87 \mathrm{~K}$ in the specific heat, indicative of a second-order phase transition [88]. By subtracting the electronic and lattice contributions to the specific heat (based on measurements of the non-magnetic reference compound $\mathrm{LuRh}_{4} \mathrm{~B}_{4}$ [101]), the magnetic entropy associated with the phase transition was determined to be $S_{M} \sim R \ln 2$, which corresponds to a ground state doublet of the $\mathrm{Sm}^{3+}$ ions.

For comparison, the upper critical field curves $H_{c 2}(T)$ for the non-magnetic compound $\mathrm{LuRh}_{4} \mathrm{~B}_{4}\left(T_{c}>11 \mathrm{~K}\right)$ and the ferromagnetic compound $\mathrm{ErRh}_{4} \mathrm{~B}_{4}\left(T_{c} \sim 8.5 \mathrm{~K}\right)$ are also shown in Fig. 11 (a) and Fig. 11 (d), respectively. For $\mathrm{LuRh}_{4} \mathrm{~B}_{4}, H_{c 2}(T)$ increases monotonically with decreasing temperature, indicating the absence of any effects on superconductivity. For the ferromagnetic compound $\mathrm{ErRh}_{4} \mathrm{~B}_{4}$, however, there is a feature at $T_{M} \sim 1.2 \mathrm{~K}$ below which $d H_{c 2} / d T$ abruptly increases, reflecting the strong pair-breaking effects associated with ferromagnetic order which act to destroy superconductivity. This is consistent with the electrical resistivity and AC susceptibility measurements displayed in Fig. 8. At higher temperatures, $H_{c 2}(T)$ is non-monotonic with a weak maximum of $H_{c 2} \sim 0.45$ $\mathrm{T}$ at $T=4.1 \mathrm{~K}$. Similar non-monotonic behavior was observed in the $H_{c 2}(T)$ curves for the antiferromagnetic superconducting compounds $\mathrm{TmRh}_{4} \mathrm{~B}_{4}$ [91] and $\mathrm{Er}_{1.2} \mathrm{Mo}_{6} \mathrm{~S}_{8}$ [77]. In the case of $\mathrm{Er}_{1.2} \mathrm{Mo}_{6} \mathrm{~S}_{8}$, this behavior has been attributed to an increase in the magnetization from the sublattice of Er ions [24], leading to increased pair-breaking.

Many of the features observed in the $H_{c 2}$ vs. $T$ data for the $\mathrm{RERh}_{4} \mathrm{~B}_{4}$ compounds discussed herein were also observed

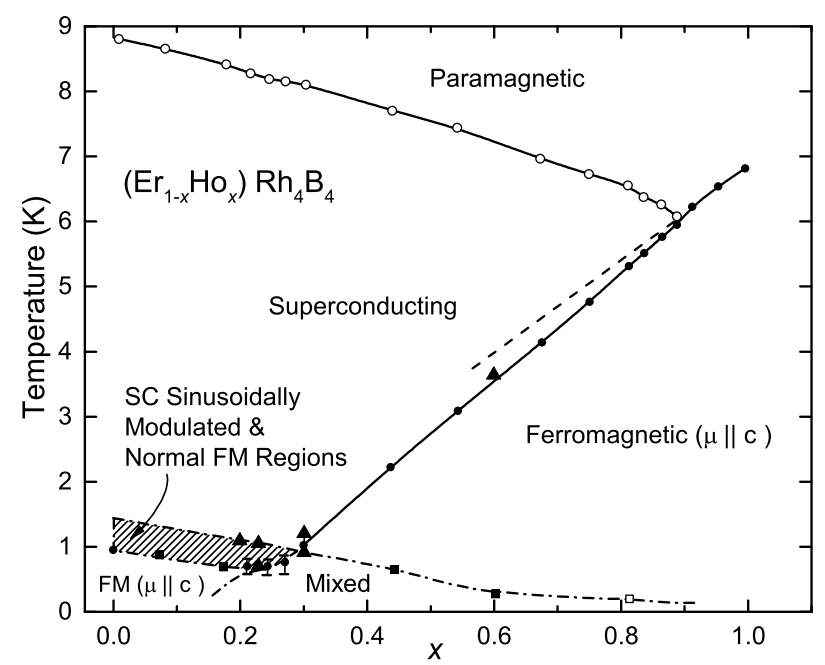

Figure 13: Low-temperature phase diagram for the $\mathrm{Er}_{1-x} \mathrm{Ho}_{x} \mathrm{Rh}_{4} \mathrm{~B}_{4}$ pseudoternary system. (from Refs. [120, 121])

in the other classes of ternary antiferromagnetic superconductors $\mathrm{RE}_{x} \mathrm{Mo}_{6} \mathrm{~S}_{8}$ and $\mathrm{RE}_{x} \mathrm{Mo}_{6} \mathrm{Se}_{8}[85,84]$. For many of these compounds, neutron-diffraction experiments in zero magnetic field have confirmed that the magnetic order appearing in the superconducting state is characterized as long-range antiferromagnetism with a correlation length greater than $300 \AA$ [114]. The anomalous behavior observed in the $H_{c 2}(T)$ data for both classes of ternary compounds appears to be a general feature associated with the antiferromagnetic transition. Multiple pairbreaking theories have been developed to account for these $H_{c 2}(T)$ data which have led to the conclusion that additional temperature-dependent pair-breaking mechanisms are responsible for the anomalies observed in these $H_{c 2}(T)$ curves [114].

\subsection{Superconductivity and Competing Magnetic Interactions in Pseudoternary Compounds}

The interplay between superconductivity and magnetic order, as well as competing types of magnetic moment anisotropy and/or magnetic order, produce rich and complex temperature composition $(T-x)$ phase diagrams in pseudoternary RE compounds. For example, two types of pseudoternary systems based on $\mathrm{RERh}_{4} \mathrm{~B}_{4}$ parent compounds have been investigated, one in which a second RE element is substituted at the RE sites and another where a different transition element is substituted at the Rh sites.

An example of a $T$ - $x$ phase diagram for the first type of pseudoternary system is shown in Fig. 13 for the $\mathrm{Er}_{1-x} \mathrm{Ho}_{x} \mathrm{Rh}_{4} \mathrm{~B}_{4}$ system [125, 120, 121, 126]. This phase diagram contains six regions: Paramagnetism (1), superconductivity (2), ferromagnetic order with the Er and Ho magnetic moments aligned within the basal plane (3) or along the tetragonal $c$-axis (4), separated by a region of mixed magnetic phases (5). In addition, there is a region above $T_{c 2}$ extending from $x=0$ to $x$ $\approx 0.3$ within which superconductivity coexists microscopically with the sinusoidally-modulated magnetic phase, first observed in $\mathrm{ErRh}_{4} \mathrm{~B}_{4}$, and macroscopically with normal ferromagnetic domains (6). 


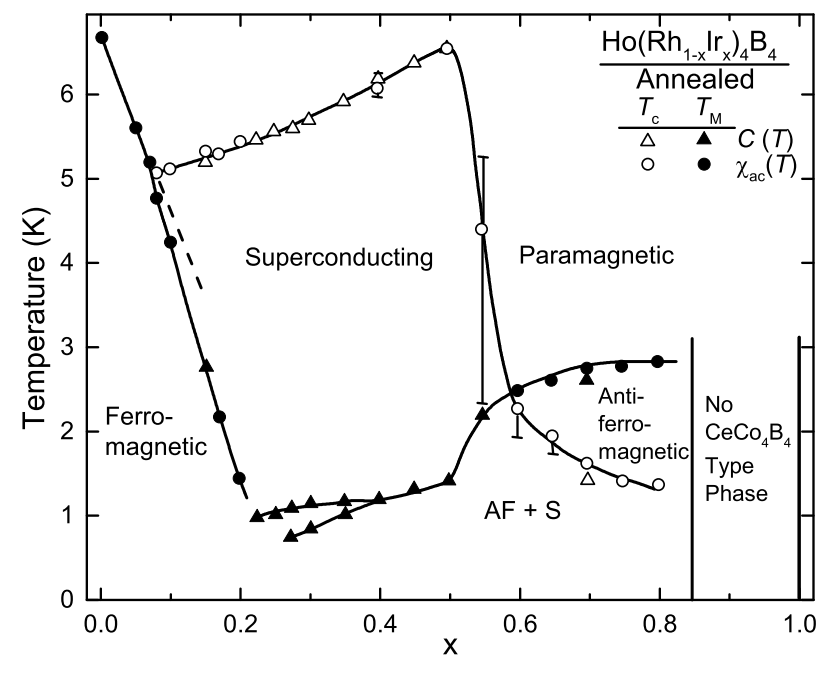

Figure 14: Low-temperature phase diagram for the $\operatorname{Ho}\left(\operatorname{Rh}_{1-x} \operatorname{Ir}_{x}\right)_{4} \mathrm{~B}_{4}$ pseudoternary system. (from Refs. [122, 123, 124])

A striking example of a $T-x$ phase diagram for the second type of pseudoternary system is shown in Fig. 14 for the $\operatorname{Ho}\left(\mathrm{Rh}_{1-x} \mathrm{Ir}_{x}\right)_{4} \mathrm{~B}_{4}$ system [127, 128, 129, 122]. The phase diagram consists of six regions: Paramagnetism (1), superconductivity (2), ferromagnetic order (3), antiferromagnetic order (4), another type of magnetic order in the range $x \approx 0.2$ to $x$ $\approx 0.4(5)$, as well as the first example of the coexistence of superconductivity and antiferromagnetic order with $T_{N}>T_{c}$ (6). Several other pseudoternary systems based on $\mathrm{RERh}_{4} \mathrm{~B}_{4}$ and $\mathrm{REMo}_{6} \mathrm{~S}_{8}$ compounds have been investigated. The reader is referred to more comprehensive reviews for additional information and references [130, 131, 125].

\subsection{Magnetic Field Induced Superconductivity}

Magnetic field induced superconductivity (MFIS) is one of the most striking phenomena produced by the interaction between superconductivity and magnetism. The mechanism responsible for this phenomenon is the exchange-field compensation effect that was proposed by Jaccarino and Peter [132] more than 20 years before MFIS was established experimentally.

The first example of MFIS, generated by the exchangefield compensation effect, was reported by Meul et al. [137] for the compound $\mathrm{Sn}_{0.25} \mathrm{Eu}_{0.75} \mathrm{Mo}_{6} \mathrm{~S}_{7.2} \mathrm{Se}_{0.8}$. The $H$ vs. $T$ phase diagram for this compound is shown in Fig. 15 with selected isotherms of normalized electrical resistance $\left(R / R_{N}\right)$ vs. applied magnetic field $H$ displayed in the inset of the figure. At the lowest temperature $(0.37 \mathrm{~K})$, the data reveal a sequence of transitions with increasing magnetic field from superconducting (S) to normal $(\mathrm{N})$ to superconducting again, and finally, back to normal (S-N-S-N). Two separate domains of superconductivity are found, one at low fields and another at high fields, whereas only one domain of superconductivity at low fields is present for a conventional superconductor.

The exchange-field compensation effect and, in turn, MFIS can occur in type-II superconductors containing magnetic moments that are antiferromagnetically coupled to the spins of the conduction electrons when the upper critical field $H_{c 2}(T)$ is determined by the paramagnetic limiting field $H_{p}$. In the $\mathrm{Eu}_{1-x} \mathrm{Sn}_{x} \mathrm{Mo}_{6} \mathrm{~S}_{8-y} \mathrm{Se}_{y}$ compounds, the magnetic moments are carried by the divalent Eu ions and their coupling to the conduction electron spins generates an "effective" magnetic field, the exchange field $H_{J}$, that acts on the conduction electron spins in the same manner as an applied magnetic field. If the sign of the coupling between the Eu magnetic moments and the conduction electron spins is negative, the direction of $H$ will be opposite to that of $H_{J}$. As a result, the effect of $H$ will be "compensated" by $H_{J}$ (see inset of Fig. 16). The sequence of S-N-S-N transitions shown in the inset of Fig. 15 can be explained in terms of the relationship between the net magnetic field $H_{T}=H-\left|H_{J}\right|$ and $H_{p}$, as illustrated schematically in Fig. 16 [136]. The upper and lower curves show the applied field $H$ and the exchange field $H_{J}$, respectively, the sum of which yields the net magnetic field $H_{T}$. The variation of $H_{T}$ with respect to the value of $H$ determines the superconducting behavior of the material.

An enhancement and anomalous temperature dependence of the upper critical field curve $H_{c 2}(T)$ due to the exchange-field compensation effect has been observed for several $\mathrm{Eu}_{1-x} \mathrm{M}_{x} \mathrm{Mo}_{6} \mathrm{~S}_{8}$ systems (where $\mathrm{M}$ is a metal such as $\mathrm{Sn}$, $\mathrm{Pb}, \mathrm{La}$ [138]) or $\mathrm{Yb}$ [139] and in $\mathrm{EuMo}_{6} \mathrm{~S}_{8}$ under pressure [140]. The $H_{c 2}(T)$ curves for EuMo ${ }_{6} \mathrm{~S}_{8}$ in its pressure-induced metallic phase are shown in Fig. 17 for several different pressures, where the solid lines are fits to the $H_{c 2}(T)$ data based on the theory of Werthamer, Helfand, Hohenberg, and Maki (WHHM) [133, 134, 135], including the exchange-field com-

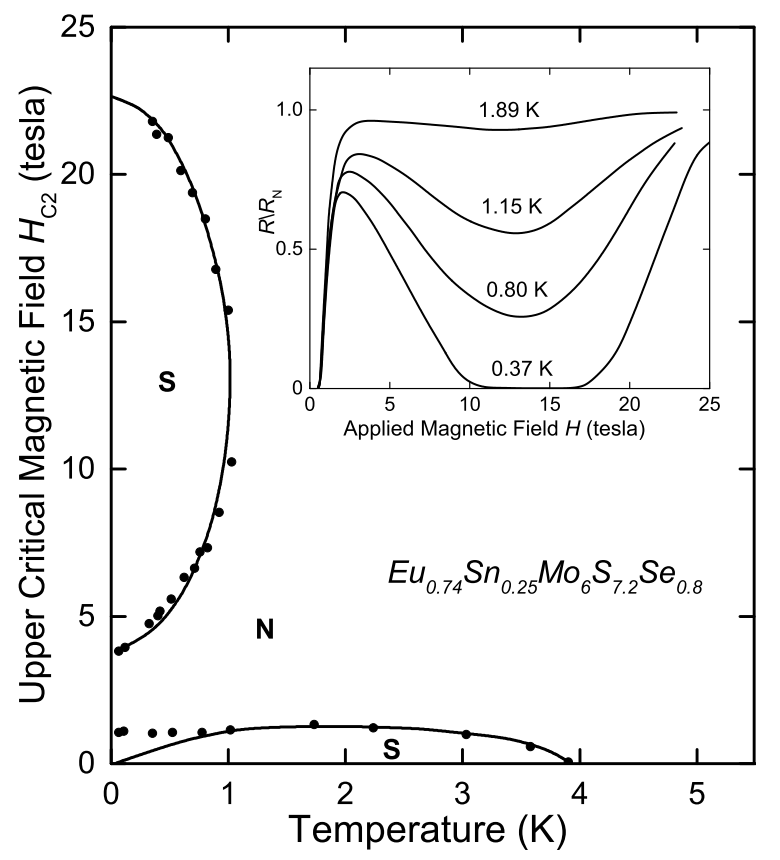

Figure 15: Resistively-determined upper critical field $H_{c 2}$ versus temperature $T$ for the compound $\mathrm{Sn}_{0.25} \mathrm{Eu}_{0.75} \mathrm{Mo}_{6} \mathrm{~S}_{7.2} \mathrm{Se}_{0.8}$. The lines are calculations of $H_{c 2}(T)$ based on the theory of Werthamer, Helfand, Hohenberg, and Maki for a conventional type-II superconductor [133, 134, 135], including the JaccarinoPeter mechanism, which have been fitted to the data. The inset shows the electrical resistance $R$, normalized to its normal state value $R_{N}$ right above $T_{c}$, versus applied magnetic field $H$ at several temperatures between $0.37 \mathrm{~K}$ and 1.89 K. (from Ref. [136]; based on data from Ref. [137]) 


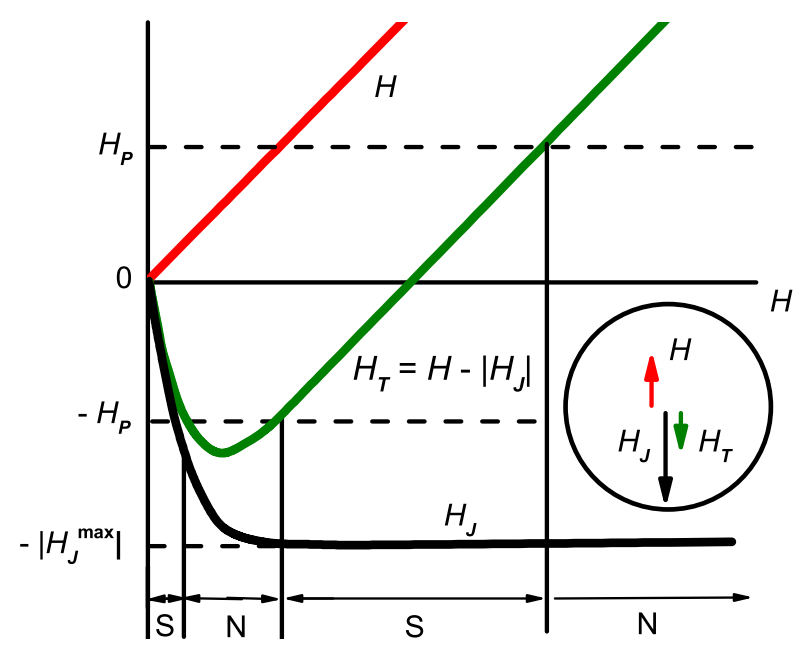

Figure 16: Schematic diagram illustrating the compensation of the applied magnetic field $H$ by the exchange field $H_{J}$ in magnetic field induced superconductivity. (from Ref. [136])

pensation effect [140]. Jaccarino and Peter originally suggested that MFIS could occur in a weakly ferromagnetic material, assuming that it would be superconducting in the absence of ferromagnetic ordering. MFIS in a ferromagnetic material remains to be discovered.

\section{Quaternary Compounds: Magnetically Ordered Super- conductors}

Shortly after the discovery of the high- $T_{c}$ cuprate superconductors, a family of "high- $T_{c}$ " RE transition metal borocarbides $\mathrm{RENi}_{2} \mathrm{~B}_{2} \mathrm{C}(\mathrm{RE}=\mathrm{Gd}, \mathrm{Tb}, \mathrm{Dy}, \mathrm{Ho}, \mathrm{Er}, \mathrm{Tm}, \mathrm{Yb}, \mathrm{Lu}, \mathrm{Y}$, and Th) was discovered in the early 1990s [10, 11, 12, 13, 14, 15, 143]. Several of the superconducting compounds in the $\mathrm{RENi}_{2} \mathrm{~B}_{2} \mathrm{C}$ series exhibit long-range magnetic order and provided a new opportunity to investigate some of the long-standing questions related to the coexistence and interplay of superconductivity and long-range magnetic order. A significant amount of research has been devoted to this class of materials and an overview of their superconducting properties may be found in another article in this special issue by Nagarajan and Mazumdar. Comprehensive treatments of the magnetic superconductors, in particular, can be found in Refs. [73, 144, 145]. Some of the phenomena discussed herein include: (1) the scaling of both $T_{c}$ and also the Néel temperature $T_{N}$ with the de Gennes factor, (2) anomalous reductions in the temperature dependence of the upper critical fields which bear a striking resemblance to the behavior observed in the upper critical fields of the ternary magnetic superconductors $\mathrm{RERh}_{4} \mathrm{~B}_{4}$ and $\mathrm{REMo}_{6} \mathrm{~S}_{8}$ (see Section 3.1.3), and (3) reentrant superconductivity as observed in $\mathrm{HoNi}_{2} \mathrm{~B}_{2} \mathrm{C}$ in low magnetic fields.

While most of the quaternary materials in the $\mathrm{RENi}_{2} \mathrm{~B}_{2} \mathrm{C}$ series exhibit magnetic order, superconductivity is found only when $\mathrm{RE}=\mathrm{Dy}, \mathrm{Ho}, \mathrm{Er}, \mathrm{Tm}, \mathrm{Lu}, \mathrm{Y}$, and Th with $T_{c}=6 \mathrm{~K}, 8 \mathrm{~K}$, $10.5 \mathrm{~K}, 11 \mathrm{~K}, 16.5 \mathrm{~K}, 15.5 \mathrm{~K}$, and $21 \mathrm{~K}$, respectively. Of these superconducting compounds, antiferromagnetic order has been
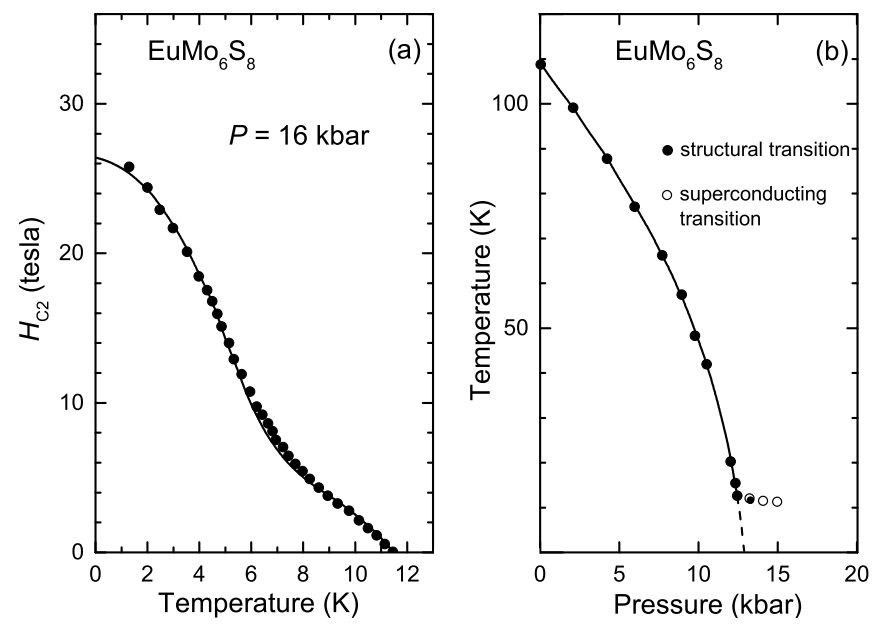

Figure 17: (a) Upper critical field $H_{c 2}$ versus temperature $T$ determined from electrical resistance measurements on $\mathrm{EuMo}_{6} \mathrm{~S}_{8}$ at a pressure of $16 \mathrm{kbar}$. The solid line is a calculation based on the theory of Werthamer, Helfand, Hohenberg, and Maki for a conventional superconductor [133, 134, 135], including the Jaccarino-Peter mechanism, which has been fitted to the data. (from Ref. $[140,141])$ (b) Structural and superconducting transition temperatures vs. pressure for $\mathrm{EuMo}_{6} \mathrm{~S}_{8}$. (from Ref. [142])

observed in $\mathrm{RENi}_{2} \mathrm{~B}_{2} \mathrm{C}\left(\mathrm{RE}=\mathrm{Dy}, \mathrm{Ho}, \mathrm{Er}\right.$, and Tm) with $T_{N}=$ $10 \mathrm{~K}$ [146, 147, 148, 149], $8 \mathrm{~K}$ [150], $6.8 \mathrm{~K}$ [151, 152], and 1.5 $\mathrm{K}[153,154]$, respectively.

These materials facilitate the study of phenomena associated with the coexistence of superconductivity and antiferromagnetic order in various temperature regimes: $T_{N}<T_{c}$ and $T_{N}>$ $T_{c}$. This relationship between $T_{c}$ and $T_{N}$ is displayed in Fig. 18 for the heavier RE compounds $\mathrm{RENi}_{2} \mathrm{~B}_{2} \mathrm{C}(\mathrm{RE}=\mathrm{Gd}, \mathrm{Tb}, \mathrm{Dy}$, Ho, Er, Tm, and Lu) in which both $T_{c}$ and $T_{N}$ are plotted as a function of the RE (upper axis) and also as a function of the de Gennes factor $(d G)$ (lower axis). Both $T_{c}$ and $T_{N}$ exhibit a linear dependence on $d G$ and the suppression of $T_{c}$ with increasing $d G$ all but vanishes at the crossover temperature from $T_{c}>T_{N}$ to $T_{c}<T_{N}$ where $T_{c}$ becomes virtually independent of $d G$ [73]. The linear dependence of $T_{N}$ on $d G$ is due to the magnetic interactions between the RE magnetic moments in these materials which are mediated by the conduction electrons via the RKKY interaction [73]. In a similar manner, the linear suppression of $T_{c}$ with increasing $d G$ across the RE series is due to the magnetic pair-breaking that results from the exchange interaction between the magnetic moments of the RE impurity ions and the spins of the conduction electrons as described by the AG theory [4, 150] (see Section 2.1.1). In the AG theory, the initial rate of depression of $T_{c}$ with increasing paramagnetic impurity concentration $x$ is proportional to $d G$. The scaling of $-\left(d T_{c} / d x\right)_{x=0}$ with $d G$ is clearly shown in Fig. 4 and the linear dependence of the suppression of $T_{c}$ can also be clearly seen from Equation 8 in Section 2.1.1.

The crossing of the ordering temperatures $T_{c}$ and $T_{N}$ that occurs between $\mathrm{HoNi}_{2} \mathrm{~B}_{2} \mathrm{C}$ and $\mathrm{DyNi}_{2} \mathrm{~B}_{2} \mathrm{C}$ is shown in the main panel of Fig. 18. This crossover from the paramagnetic regime $\left(T_{c}>T_{N}\right)$ to the magnetically ordered regime $\left(T_{c}<\right.$ $T_{N}$ ) provided the motivation for a study of pseudoquaternary $\mathrm{Ho}_{1-x} \mathrm{Dy}_{x} \mathrm{Ni}_{2} \mathrm{~B}_{2} \mathrm{C}[73,147,148]$. The data for $T_{c}$ (small red 
circles) and $T_{N}$ (small blue squares) between $\mathrm{HoNi}_{2} \mathrm{~B}_{2} \mathrm{C}$ and DyNi $_{2} \mathrm{~B}_{2} \mathrm{C}$ indicate that the suppression of $T_{c}$ vanishes at the crossover to the magnetically ordered state $\left(T_{c}<T_{N}\right)$. This intriguing behavior motivated a similar study involving the pseudoquaternary series $\mathrm{Lu}_{1-x} \mathrm{Dy}_{x} \mathrm{Ni}_{2} \mathrm{~B}_{2} \mathrm{C}$ in which the nonmagnetic species Lu plays the role that the magnetic species Ho played in the study of $\mathrm{Ho}_{1-x} \mathrm{Dy}_{x} \mathrm{Ni}_{2} \mathrm{~B}_{2} \mathrm{C}$ [155]. Again, there is an obvious suppression of $T_{c}$ (seen on the left side of the graph in the inset of Fig. 18) as Lu ions are replaced by magnetic Dy ions in $\mathrm{LuNi}_{2} \mathrm{~B}_{2} \mathrm{C}$. There is no long-range antiferromagnetic order for low concentrations of Dy impurities; the suppression of $T_{c}$ reflects the magnetic pair-breaking that occurs via the exchange interaction between the localized magnetic moments of the Dy impurity ions and the conduction electrons in $\mathrm{LuNi}_{2} \mathrm{~B}_{2} \mathrm{C}$.

On the right side of the inset of Fig. 18, there is also a rapid suppression of $T_{c}$ for higher Dy concentrations in $\mathrm{Lu}_{1-x} \mathrm{Dy}_{x} \mathrm{Ni}_{2} \mathrm{~B}_{2} \mathrm{C}$ where $T_{c}<T_{N}$. Here, the magnetically ordered regime applies as $T_{c}<T_{N}$. Both superconductivity and the long-range antiferromagnetic order associated with the Dy sublattice in $\mathrm{Lu}_{1-x} \mathrm{Dy}_{x} \mathrm{Ni}_{2} \mathrm{~B}_{2} \mathrm{C}$ are suppressed with increasing concentration of non-magnetic Lu. The suppression of $T_{N}$ is driven by the random substitution of non-magnetic Lu for magnetic Dy in the RE sublattice which has a disruptive effect on the RKKY modes. In the magnetically ordered regime, the suppression of $T_{c}$ with increasing concentration of nonmagnetic $\mathrm{Lu}$ is more surprising. This response may be understood if one considers the effect that the nonmagnetic Lu ions have on the RKKY interactions between the magnetic Dy ions. By introducing nonmagnetic Lu ions into the Dy sublattice, the correlations between Dy ions are weakened on average and the Dy ions are more likely to behave as localized magnetic moments that can break up pairs of superconducting electrons via the exchange interaction. The results for $\mathrm{Lu}_{1-x} \mathrm{Dy}_{x} \mathrm{Ni}_{2} \mathrm{~B}_{2} \mathrm{C}$ confirm the existence of magnetic pair-breaking via the exchange interaction in the paramagnetic state and suggest the same pairbreaking mechanism can affect superconductivity in the magnetically ordered state.

\subsection{Upper Critical Fields of Rare Earth Borocarbide Super- conductors}

Upper critical field measurements of various members of the quaternary borocarbides provide additional means to investigate the effects of local moment RE magnetism on superconductivity. The upper critical field $H_{c 2}(T)$ curves for the antiferromagnetic superconductors $\mathrm{RENi}_{2} \mathrm{~B}_{2} \mathrm{C}(\mathrm{RE}=\mathrm{Ho}, \mathrm{Er}, \mathrm{Tm})$ are shown in Fig. 19 where $T_{c}>T_{N}$ for each compound [150]. For a discussion of $\mathrm{DyNi}_{2} \mathrm{~B}_{2} \mathrm{C}$ where $T_{c}<T_{N}$, see the previous section. In each of these compounds, there is a noticeable downturn in the $H_{c 2}(T)$ curves as shown in Fig. 19. The upper critical field $H_{c 2}(T)$ curves for the non-magnetic superconductors $\mathrm{LuNi}_{2} \mathrm{~B}_{2} \mathrm{C}$ and $\mathrm{YNi}_{2} \mathrm{~B}_{2} \mathrm{C}$ are shown for comparison and are illustrative for the absence of anomalous features in their temperature dependence. There is an overall suppression of $H_{c 2}(T)$ that tracks with the suppression of $T_{c}$ across the series $\mathrm{RENi}_{2} \mathrm{~B}_{2} \mathrm{C}(\mathrm{RE}=\mathrm{Ho}, \mathrm{Er}, \mathrm{Tm}, \mathrm{Y}$ and $\mathrm{Lu})[150]$; a similar relation between $H_{c 2}(T)$ and $T_{c}$ exists among the ternary magnetic

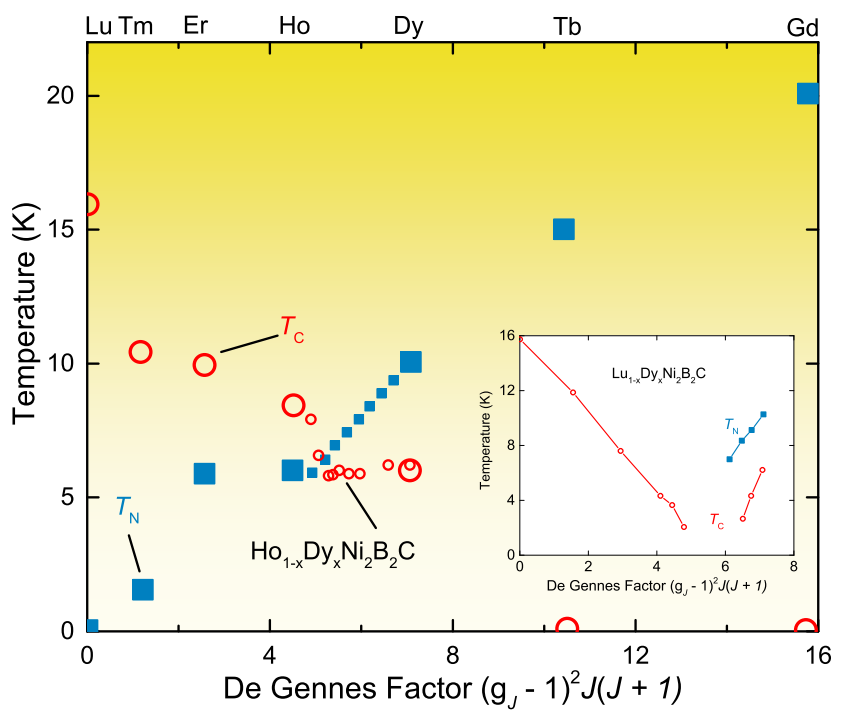

Figure 18: Superconducting transition temperatures $T_{C}$ (red circles) and Néel temperatures $T_{N}$ (blue squares) plotted as a function of the de Gennes factor for various $\mathrm{RENi}_{2} \mathrm{~B}_{2} \mathrm{C}$ compounds with the $\mathrm{RE}$ indicated in the upper axis. The smaller symbols represent data for the pseudoquaternary compounds $\mathrm{Ho}_{1-x} \mathrm{Dy}_{x} \mathrm{Ni}_{2} \mathrm{~B}_{2} \mathrm{C}$. The inset shows $T_{c}$ (red symbols) and Néel temperatures $T_{N}$ (blue symbols) plotted as a function of the average de Gennes factor for the pseudoquaternary compounds $\mathrm{Lu}_{1-x} \mathrm{Dy}_{x} \mathrm{Ni}_{2} \mathrm{~B}_{2} \mathrm{C}$. (from Refs. [73])

superconductors (see Fig. 11 in Section 3.1.3).

The broad peak in $H_{c 2}(T)$ for $\mathrm{TmNi}_{2} \mathrm{~B}_{2} \mathrm{C}$ is similar to those observed in the $H_{c 2}(T)$ curves for several of the ternary magnetic superconductors including the ferromagnetic superconductor $\mathrm{ErRh}_{4} \mathrm{~B}_{4}$ as shown in Fig. 11 and the antiferromagnetic superconducting compounds $\mathrm{TmRh}_{4} \mathrm{~B}_{4}$ [91] and $\mathrm{Er}_{1.2} \mathrm{Mo}_{6} \mathrm{~S}_{8}$ [77] (see Section 3.1.3). The maxima in the $H_{c 2}(T)$ curves occur at temperatures well above the magnetic ordering temperatures and correspond to the magnetization from the sublattice of $\mathrm{RE}$ ions which increases with increasing $\boldsymbol{H}_{\boldsymbol{T}}$ via the Brillouin function and acts in concert with the applied magnetic field $\boldsymbol{H}$ as a superconducting electron pair-breaker [131, 156].

$\mathrm{TmNi}_{2} \mathrm{~B}_{2} \mathrm{C}$ is distinct from the other magnetic superconductors in that the $\mathrm{Tm}^{3+}$ moments align parallel along the $c$ axis. For a discussion regarding the anisotropy observed in the RE sublattice magnetization for single crystals of $\mathrm{RENi}_{2} \mathrm{~B}_{2} \mathrm{C}$ compounds, the reader is referred to the neutron-diffraction study on the $\mathrm{RENi}_{2} \mathrm{~B}_{2} \mathrm{C}$ series by Lynn et al. [157]. This anisotropy in the Tm sublattice magnetization is reflected in the difference in the overall magnitude of $H_{c 2}(T)$ which is smaller by a factor of 2 when $\boldsymbol{H} \| \boldsymbol{c}$ compared to when $\boldsymbol{H} \perp \boldsymbol{c}$ (not shown in Fig. 19) [73].

The local minimum observed in $H_{c 2}(T)$ near $T_{N}=5.8 \mathrm{~K}$ for $\mathrm{ErNi}_{2} \mathrm{~B}_{2} \mathrm{C}$ as shown in Fig. 19 occurs only when $\boldsymbol{H} \| \boldsymbol{c}$. The $H_{c 2}(T)$ curve for the case when $\boldsymbol{H} \perp \boldsymbol{c}$ (not shown) exhibits an inflection point at $T_{N}$ [73]. This suggests that any increase in the magnetic pair-breaking that takes place near the antiferromagnetic ordering temperature is sensitive to the orientation of the magnetic moments of the Er atoms within the Er sublattice. Some of the theoretical work that emerged from the intense 


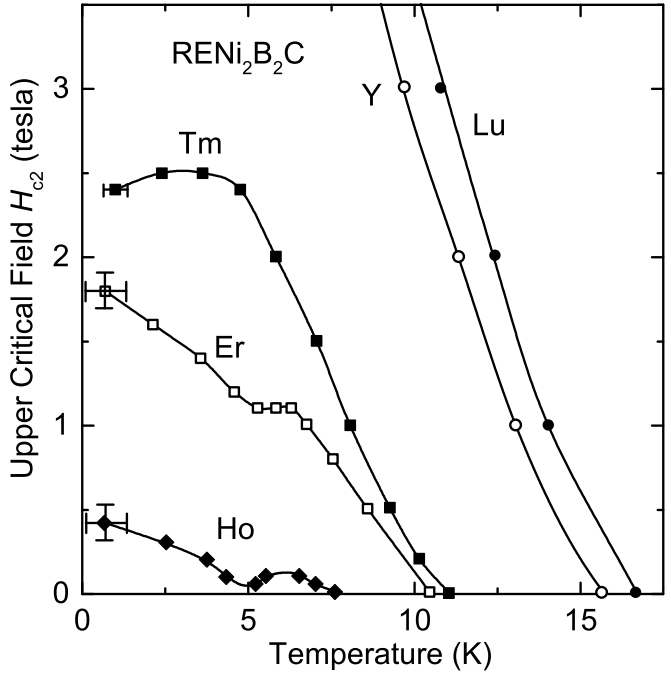

Figure 19: The upper critical field $H_{c 2}(T)$ for the $\mathrm{RENi}_{2} \mathrm{~B}_{2} \mathrm{C}(\mathrm{RE}=\mathrm{Lu}, \mathrm{Y}, \mathrm{Tm}$, Er, Ho) compounds. The superconducting transition temperatures $T_{c}$ at each field were determined from electrical resistivity measurements (not shown); the midpoint of the resistive transitions were used to define $T_{c}$. (from Ref. [150])

study of the ternary magnetic superconductors suggested that the pair-breaking mechanisms responsible for the small downturns in $H_{c 2}(T)$ would most likely include a dependence on temperature [114]. The exact nature of the pair-breaking mechanism responsible for the small reductions in $H_{c 2}(T)$ observed in such close proximity to the Néel temperatures $[131,150]$ is still unclear. Other predictions for an increase in pair-breaking in magnetic superconductors near $T_{N}$ are based on a generalization of AG theory in the limit of a large concentration of magnetic ions that form a regular lattice [158] (see Section 2.1.1 for a discussion of pair-breaking and AG theory in the dilute limit). The theory maintains that the wavevector $\boldsymbol{q}_{\boldsymbol{m}}$ that characterizes the magnetic ordering of the RE sublattice (or spindensity wave) is the same wavevector $\boldsymbol{q}$ that yields a maximum in the magnetic susceptibility $\chi(\boldsymbol{q})[156,158]$. Neutrondiffraction experiments have confirmed that the $\mathrm{Er}^{3+}$ moments of the Er sublattice are all aligned in the basal plane along the crystallographic [010] direction (i.e., perpendicular to the $c$ axis) [157]. These Er magnetic moments form a transversely polarized spin-density wavevector in the crystallographic [100] direction which is indeed a magnetic wavevector that fulfills the criterion in the theory for increased pair-breaking near $T_{N}$ $[157,156,158]$.

\subsection{Reentrant Superconductivity in $\mathrm{HoNi}_{2} \mathrm{~B}_{2} \mathrm{C}$}

As is shown in Fig. 19, the $H_{c 2}(T)$ curve for $\mathrm{HoNi}_{2} \mathrm{~B}_{2} \mathrm{C}$ is qualitatively similar to the $H_{c 2}(T)$ curve for $\mathrm{ErNi}_{2} \mathrm{~B}_{2} \mathrm{C}$, but the downturn in $H_{c 2}(T)$ for $\mathrm{HoNi}_{2} \mathrm{~B}_{2} \mathrm{C}$ is much deeper than that observed for $\mathrm{ErNi}_{2} \mathrm{~B}_{2} \mathrm{C}$ which is indicative of a return to the normal state at $T_{N}=5 \mathrm{~K}$. There is a second superconducting transition which is reflected in a steady and monotonic increase in the upper critical field as the temperature decreases to $0 \mathrm{~K}$.

The compound $\mathrm{HoNi}_{2} \mathrm{~B}_{2} \mathrm{C}$ is one of the few magnetic superconductors that exhibits reentrant superconductivity and is the only known antiferromagnetic superconductor to exhibit reentrant behavior in zero field [150]. Interestingly, the long-range magnetic order observed in $\mathrm{HoNi}_{2} \mathrm{~B}_{2} \mathrm{C}$ is responsible for both the destruction of superconductivity at $T_{N}=5 \mathrm{~K}$ as well as the restoration of superconductivity at lower temperatures $T<5$ $\mathrm{K}$. The antiferromagnetic order at the higher temperature $T_{N}$ $=5 \mathrm{~K}$ is incommensurate and is incompatible with superconductivity; at lower temperatures, the antiferromagnetic structure becomes commensurate, allowing superconductivity to reemerge. This antiferromagnetic order continues to coexist with superconductivity at low temperatures. Similar behavior was previously observed for the ternary magnetic superconductor $\mathrm{NdRh}_{4} \mathrm{~B}_{4}$ as shown in Fig. 11 (b) in Section 3.1.3. In that compound, there are two magnetic ordering temperatures, both of which involve $\mathrm{Nd}^{3+}$ moments that align along the $c$ axis. The antiferromagnetic transition at higher temperature, however, is characterized by a sinusoidal modulation in the magnetic moment along the crystallographic [110] direction which competes with superconductivity. Eventually this type of order gives way to a sinusoidal modulation in the magnetic moment along the crystallographic [100] direction which is more compatible with superconductivity.

\section{Concluding Remarks}

Early research on the interplay between superconductivity and magnetism was largely driven by the question of whether there could be coexistence between superconductivity and longrange magnetic order. In an attempt to answer this question, systematic investigations of the effect of paramagnetic impurities dissolved into a superconducting host led to the discovery of some new and exotic physics. Binary and pseudobinary superconducting compounds containing small amounts of RE magnetic impurities at best exhibited short-range "glassy" magnetic order; i.e, there was no observed long-range magnetic order associated with a sublattice of magnetic ions in these materials. The magnetic exchange interaction between the localized moment of the impurity ion and spins of the conduction electrons (even at dilute impurity concentrations) had a destructive effect on superconductivity in these compounds; however, the presence of such impurities produced some interesting phenomena such as gapless superconductivity, manybody Kondo physics, reentrant superconductivity, temperature dependent pair-breaking, etc. Many of the phenomena were successfully explained with rigorous and quantitative theories and, quite remarkably, some of the results were predicted long before they were realized in experiments.

In the 1970s, the idea that a weak magnetic exchange interaction might allow superconductivity to coexist with long-range magnetic order led to the discovery of two classes of ternary magnetic superconducting compounds: (1) the rhodium borides and (2) the molybdenum chalcogenides (Chevrel phases). These compounds provided physicists with an opportunity to explore the phenomena that emerge from the coexistence of superconductivity and long-range magnetic order including: reentrant superconductivity in ferromagnetic and antiferromagnetic 
compounds; the generation of a sinusoidally-modulated magnetic state with a wavelength $\sim 100 \AA$ that coexists with superconductivity in a narrow range of temperature above the reentrant transition at $T_{c 2}$ in ferromagnetic superconductors; the exchange-field compensation effect observed in antiferromagnetic compounds as manifested in MFIS; and the anomalous reductions in the upper critical field curves in the magnetic state. Many of these phenomena were also studied in the 1990s with the serendipitous discovery and systematic investigations of the quaternary borocarbide magnetic superconductors.

The phenomena discussed herein are by no means an exhaustive treatment of the exotic physics observed within compounds containing RE or actinide elements with partially-filled $f$-electron shells. Heavy-fermion materials, for example, can exhibit superconducting and magnetic ground states or the coexistence of both. These $f$-electron materials provide additional opportunities to investigate the interplay between superconductivity and magnetic order. Interestingly, the degree of hybridization between the $f$-electron and conduction-electron states that underlies the behavior of the conventional superconductors discussed in this article also plays a central role in the phenomena observed in heavy-fermion compounds; however, these heavy-electron materials exhibit unconventional superconductivity (see the dedicated article on heavy-fermion superconductivity in this special issue). As a final comment, we note that the study of conventional magnetic superconductors over the last 60 years has provided a framework for the study and development of our understanding of unconventional superconductivity in the heavy-fermion compounds, the cuprates, and other classes of materials in which superconductivity is in proximity to, or coexists with, a magnetically-ordered phase.

\section{Acknowledgements}

Work at the University of California, San Diego was performed with financial support from the U.S. Department of Energy, Office of Basic Energy Sciences, Division of Materials Sciences and Engineering under Award Grant No. DE-FG0204-ER46105, the National Science Foundation under Grant No. DMR-1206553, and the National Nuclear Security Administration under the Stewardship Science Academic Alliance program through the U.S. Department of Energy Grant No. DENA0001841.

[1] V. L. Ginzburg, Ferromagnetic superconductors, Soviet Physics JETP 4 (1957) 153-160.

[2] B. T. Matthias, H. Suhl, E. Corenzwit, Spin exchange in superconductors, Phys. Rev. Lett. 1 (1958) 92-94.

[3] B. T. Matthias, H. Suhl, E. Corenzwit, Ferromagnetic superconductors, Phys. Rev. Lett. 1 (1958) 449-450.

[4] A. A. Abrikosov, L. P. Gor'kov, Contribution to the theory of superconducting alloys with paramagnetic impurities, Soviet Physics JETP 12 (1961) 1243-1253.

[5] M. B. Maple, Paramagnetic Impurities in Superconductors, in: G. T. Rado, H. Suhl (Eds.), Magnetism Volume 5: Magnetic Properties of Metallic Alloys, Academic Press, New York and London, 1973, pp. 289-326.

[6] M. B. Maple, Superconductivity: A probe of the magnetic state of local moments in metals, Appl. Phys. 9 (1976) 179-204.

[7] R. Chevrel, M. Sergent, J. Prigent, Sur de nouvelles phases sulfurées ternaires du molybdéne, J. Solid State Chem. 3 (1971) 515 - 519.
[8] B. T. Matthias, M. Marezio, E. Corenzwit, A. S. Cooper, H. E. Barz, High-temperature superconductors, the first ternary system, Science 175 (1972) 1465-1466.

[9] B. T. Matthias, E. Corenzwit, J. M. Vandenberg, H. E. Barz, High superconducting transition temperatures of new rare earth ternary borides, Proc. National Acad. Sci. 74 (1977) 1334-1335.

[10] C. Mazumdar, R. Nagarajan, C. Godart, L. Gupta, M. Latroche, S. Dhar, C. Levy-Clement, B. Padalia, R. Vijayaraghavan, Superconductivity at $12 \mathrm{~K}$ in Y-Ni-B system, Solid State Comm. 87 (1993) $413-416$.

[11] R. Nagarajan, C. Mazumdar, Z. Hossain, S. K. Dhar, K. V. Gopalakrishnan, L. C. Gupta, C. Godart, B. D. Padalia, R. Vijayaraghavan, Bulk superconductivity at an elevated temperature $T_{c} \approx 12 \mathrm{~K}$ in a nickel containing alloy system Y-Ni-B-C, Phys. Rev. Lett. 72 (1994) 274-277.

[12] C. Mazumdar, R. Nagarajan, C. Godart, L. Gupta, M. Latroche, S. Dhar, C. Levy-Clement, B. Padalia, R. Vijayaraghavan, Superconductivity at 12 K in Y-Ni-B system, Physica B 194 -196 (1994) 1985 - 1986.

[13] R. J. Cava, H. Takagi, B. Batlogg, H. W. Zandbergen, J. J. Krajewski, W. F. Peck, R. B. van Dover, R. J. Felder, T. Siegrist, K. Mizuhashi, J. O. Lee, H. Eisaki, S. A. Carter, S. Uchida, Superconductivity at $23 \mathrm{~K}$ in yttrium palladium boride carbide, Nature 367 (1994) 146-148.

[14] R. J. Cava, H. Takagi, H. W. Zandbergen, J. J. Krajewski, W. F. Peck, T. Siegrist, B. Batlogg, R. B. van Dover, R. J. Felder, K. Mizuhashi, J. O. Lee, H. Eisaki, S. Uchida, Superconductivity in the quartenary inter-metallic compounds $\mathrm{LuNi}_{2} \mathrm{~B}_{2} \mathrm{C}$, Nature 367 (1994) 252-253.

[15] T. Siegrist, H. W. Zandbergen, R. J. Cava, J. J. Krajewski, W. F. Peck, The crystal structure of superconducting $\mathrm{LuNi}_{2} \mathrm{~B}_{2} \mathrm{C}$ and the related phase LuNiBC, Nature 367 (1994) 254-256.

[16] M. B. Maple, The superconducting transition temperature of $\mathrm{La}_{1-x} \mathrm{Gd}_{x} \mathrm{Al}_{2}$, Phys. Lett. A 26 (1968) $513-514$.

[17] J. Bardeen, L. N. Cooper, J. R. Schrieffer, Theory of superconductivity, Phys. Rev. 108 (1957) 1175-1204.

[18] S. Skalski, O. Betbeder-Matibet, P. R. Weiss, Properties of superconducting alloys containing paramagnetic impurities, Phys. Rev. 136 (1964) A1500-A1518.

[19] J. Jensen, A. R. Mackintosh, Rare Earth Magnetism: Structures and Excitations, The International Series of Monographs on Physics, Clarendon Press, Oxford, 1991.

[20] M. B. Maple, E. D. Bauer, V. S. Zapf, J. Wosnitza, Unconventional Superconductivity in Novel Materials, in: K. H. Bennemann, J. B. Ketterson (Eds.), Superconductivity, Springer Berlin Heidelberg, 2008, pp. 639-762.

[21] R. D. Parks, Experimental Studies of Superconductors with Magnetic Impurities, in: P. R. Wallace (Ed.), Superconductivity, Vol. 2, Gordon And Breach, New York, 1966, p. 623.

[22] B. T. Matthias, H. Suhl, E. Corenzwit, Further experiments concerning the spin-electron interactions in superconductors, J. Phys. Chem. Solids 13 (1960) $156-159$.

[23] M. B. Maple, Coexistence of Superconductivity and Magnetism, in: B. Deaver, J. Ruvalds (Eds.), Advances in Superconductivity, Plenum Press, New York and London, 1983, pp. 279-346.

[24] M. B. Maple, H. C. Hamaker, L. D. Woolf, Superconductivity, magnetism and their mutual interaction in ternary rare earth rhodium borides and some ternary rare earth transition metal stannides, in: M. B. Maple, $\emptyset$. Fischer (Eds.), Superconductivity in Ternary Compounds 2, SpringerVerlag, Berlin Heidelberg New York, 1982, pp. $99-141$.

[25] Z. Fisk, B. T. Matthias, E. Corenzwit, Rare earth impurities in $\mathrm{YB}_{6}$ and $\mathrm{ZrB}_{12}$, Proc. National Acad. Sci. 64 (1969) 1151-1154.

[26] L. E. De Long, R. W. McCallum, W. A. Fertig, M. B. Maple, J. G. Huber, Observation of a Kondo effect for a dilute alloy containing Sm impurities, Solid State Comm. 22 (1977) 245 - 249.

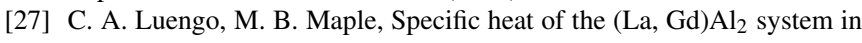
the superconducting and normal state, Solid State Comm. 12 (1973) 757 -762 .

[28] W. R. Decker, D. K. Finnemore, Critical-field curves for gapless superconductors, Phys. Rev. 172 (1968) 430-436.

[29] F. Reif, M. A. Woolf, Energy gap in superconductors containing paramagnetic impurities, Phys. Rev. Lett. 9 (1962) 315-316.

[30] M. A. Woolf, F. Reif, Effect of magnetic impurities on the density of states of superconductors, Phys. Rev. 137 (1965) A557-A564.

[31] D. K. Finnemore, D. L. Johnson, J. E. Ostenson, F. H. Spedding, B. J. Beaudry, Superconductivity in Pure La and La-Gd, Phys. Rev. 137 
(1965) A550-A556.

[32] L. P. Gor'kov, A. I. Rusinov, Contribution to the theory of superconducting alloys with paramagnetic impurities, Soviet Physics JETP 19 (1964) 922-931.

[33] A. I. Rusinov, On the theory of gapless superconductivity in alloys containing paramagnetic impurities, Soviet Physics JETP 29 (1969) 11011106.

[34] J. Friedel, XIV. The distribution of electrons around impurities in monovalent metals, Phil. Mag. 43 (1952) 153-189.

[35] P. W. Anderson, Localized magnetic states in metals, Phys. Rev. 124 (1961) 41-53.

[36] J. R. Schrieffer, P. A. Wolff, Relation between the Anderson and Kondo Hamiltonians, Phys. Rev. 149 (1966) 491-492.

[37] J. Kondo, g-shift and anomalous Hall effect in gadolinium metals, Prog. Theor. Phys. 28 (1962) 846-856.

[38] P. G. De Gennes, Polarisation de charge (ou de spin) au voisinage d'une impureté dans un alliage, J. Phys. Radium 23 (1962) 630-636.

[39] J. Kondo, Resistance minimum in dilute magnetic alloys, Prog. Theor. Phys. 32 (1964) 37-49.

[40] W. Felsch, K. Winzer, G. v. Minnigerode, Magnetic susceptibility of (La,Ce)Al $\mathrm{Al}_{2}$ alloys, Z. Phys. B Condens. Matter 21 (1975) 151-156.

[41] A. Benoit, J. Flouquet, J. Sanchez, Nuclear orientation experiments on $\mathrm{Al}_{2}$ (La,Ce) alloys, Solid State Comm. 13 (1973) 1581 - 1583.

[42] T. Sugawara, H. Eguchi, Note on the Kondo temperature of La-Ce alloys, J. Phys. Soc. Jpn. 26 (1969) 1322-1322.

[43] K. S. Kim, M. B. Maple, Kondo effect in $\mathrm{La}_{1-x} \mathrm{Ce}_{x}$ alloys under pressure, Phys. Rev. B 2 (1970) 4696-4699.

[44] W. Gey, E. Umlauf, Pressure dependence of the Kondo effect in La-Ce alloys, Z. Phys. B Condens. Matter 242 (1971) 241.

[45] J. Flouquet, Nuclear-orientation studies of LaCe, AuCe, and AgCe, Phys. Rev. Lett. 27 (1971) 515-517.

[46] M. B. Maple, Superconductivity and magnetism of lanthanum-rare earth dialuminides, Ph.D. thesis, University of California, San Diego (1969).

[47] M. B. Maple, Z. Fisk, Proceedings of the 11th international conference on low temperature physics, in: J. F. Allen, D. M. Findlayson, D. M. McCall (Eds.), Proceedings of the 11th International Conference on Low Temperature Physics, Vol. 2, St. Andrews, Scotland, 1968, pp. 12881292.

[48] J. A. White, H. J. Williams, J. H. Wernick, R. C. Sherwood, Observation of a possible large crystalline Stark splitting in metallic Ce: $\mathrm{LaAl}_{2}$, Phys. Rev. 131 (1963) 1039-1042.

[49] P. Fulde, L. L. Hirst, A. Luther, Superconductors containing impurities with crystal-field split energy levels, Z. Phys. 230 (1970) 155 - 177.

[50] M. B. Maple, W. A. Fertig, A. C. Mota, L. E. DeLong, D. Wohlleben, R. Fitzgerald, The re-entrant superconducting-normal phase boundary of the Kondo system (La, Gd) $\mathrm{Al}_{2}$, Solid State Comm. 11 (1972) $829-$ 834 .

[51] E. Müller-Hartmann, J. Zittartz, Kondo effect in superconductors, Phys. Rev. Lett. 26 (1971) 428-432.

[52] E. Müller-Hartmann, Recent Theoretical Work on Magnetic Impurities in Superconductors, in: G. T. Rado, H. Suhl (Eds.), Magnetism Volume 5: Magnetic Properties of Metallic Alloys, Academic Press, New York and London, 1973, pp. 353 - 382.

[53] P. Schlottmann, Transition temperature and specific heat discontinuity for superconducting alloys containing Kondo impurities, Solid State Comm. 16 (1975) 1297 - 1300.

[54] P. Schlottmann, On properties of superconducting alloys containing Kondo impurities, J. Low Temp. Phys. 20 (1975) 123-154.

[55] A. Ludwig, M. J. Zuckermann, The dependence of the superconducting critical temperature of dilute magnetic alloys on impurity concentration, J. Phys. F: Metal Phys 1 (1971) 516.

[56] E. Müller-Hartmann, J. Zittartz, Theory of magnetic impurities in superconductors II, Z. Phys. 234 (1970) 58-69.

[57] M. B. Maple, J. G. Huber, B. R. Coles, A. C. Lawson, Superconductivity and normal-state properties of ThU alloys, J. Low Temp. Phys. 3 (1970) 137-145.

[58] G. Riblet, K. Winzer, Vanishing of superconductivity below a second transition temperature in $\left(\mathrm{La}_{1-x} \mathrm{Ce}_{x}\right) \mathrm{Al}_{2}$ alloys due to the Kondo effect, Solid State Comm. 9 (1971) 1663 - 1665

[59] J. Huber, W. Fertig, M. Maple, Superconducting-normal phase boundaries of (La, Th)Ce systems, Solid State Comm. 15 (1974) 453 - 457.
[60] M. B. Maple, K.-S. Kim, Pressure-dependent pair breaking in superconducting $\mathrm{La}_{3-x} \mathrm{Ce}_{x}$ in alloys, Phys. Rev. Lett. 23 (1969) 118-120.

[61] M. B. Maple, J. Wittig, K. S. Kim, Pressure-induced magneticnonmagnetic transition of Ce impurities in La, Phys. Rev. Lett. 23 (1969) 1375-1377.

[62] M. Maple, T. Smith, The effect of pressure on the superconducting transition temperature of $\mathrm{La}_{1-x} \mathrm{Ce}_{x} \mathrm{Al}_{2}$ and $\mathrm{La}_{1-x} \mathrm{Gd}_{x} \mathrm{Al}_{2}$ alloys, Solid State Comm. 7 (1969) 515 - 517.

[63] J. G. Huber, J. Brooks, D. Wohlleben, M. B. Maple, Magnetic susceptibility study of the continuous demagnetization of Ce impurities in the (La,Th)Ce system, AIP Conf. Proc. 24 (1975) 475-476.

[64] S. Ortega, M. Roth, C. Rizzuto, M. Maple, Magnetic to nonmagnetic transition of cerium impurities in (La, Th) alloys, Solid State Comm. 13 (1973) $5-7$.

[65] C. A. Luengo, J. G. Huber, M. B. Maple, M. Roth, Calorimetric investigation of the continuous demagnetization of Ce impurities in superconducting La, Th alloys, Phys. Rev. Lett. 32 (1974) 54-58.

[66] C. A. Luengo, J. G. Huber, M. B. Maple, M. Roth, Demagnetization of Ce impurities in superconducting (La, Th)Ce alloys: Specific heat results, J. Low Temp. Phys. 21 (1975) 129-151.

[67] A. B. Kaiser, Effect of non-magnetic localized states in superconducting alloys, J. Phys. C: Solid State Phys. 3 (1970) 410.

[68] J. G. Huber, M. B. Maple, The effect of nonmagnetic $4 f$ resonances on the superconducting transition temperature: ThCe alloys, J. Low Temp. Phys. 3 (1970) 537-544.

[69] J. Huber, M. Maple, Superconducting-normal phase boundary of superconductors containing nearly nonmagnetic impurities, Solid State Comm. 8 (1970) 1987 - 1989.

[70] H. Suhl, B. T. Matthias, E. Corenzwit, Some further results on ferromagnetism in relation to superconductivity, J. Phys. Chem. Solids 11 (1959) $346-348$.

[71] $\varnothing$. Fischer, M. Peter, Recent Work on Ferromagnetic Superconductors, in: G. T. Rado, H. Suhl (Eds.), Magnetism Volume 5: Magnetic Properties of Metallic Alloys, Academic Press, New York and London, 1973, pp. 327-352.

[72] S. Roth, Coexistence of superconductivity and magnetic order, Appl. Phys. 15 (1978) 1-11.

[73] P. C. Canfield, P. L. Gammel, D. J. Bishop, New magnetic superconductors: A toy box for solid-state physicists, Phys. Today 51 (1998) 40-46.

[74] J. Remeika, G. Espinosa, A. Cooper, H. Barz, J. Rowell, D. McWhan, J. Vandenberg, D. Moncton, Z. Fisk, L. Woolf, H. Hamaker, M. Maple, G. Shirane, W. Thomlinson, A new family of ternary intermetallic superconducting/magnetic stannides, Solid State Comm. 34 (1980) 923 926.

[75] Ø. Fischer, A. Treyvaud, R. Chevrel, M. Sergent, Superconductivity in the $\mathrm{Re}_{x} \mathrm{Mo}_{6} \mathrm{~S}_{8}$, Solid State Comm. 17 (1975) $721-724$

[76] R. Shelton, R. McCallum, H. Adrian, Superconductivity in rare earth molybdenum selenides, Phys. Lett. A 56 (1976) $213-214$.

[77] M. Ishikawa, $\varnothing$. Fischer, Magnetic ordering in the superconducting state of rare earth molybdenum sulphides, $(\mathrm{RE})_{1.2} \mathrm{Mo}_{6} \mathrm{~S}_{8}(\mathrm{RE}=\mathrm{Tb}$, Dy and Er), Solid State Comm. 24 (1977) 747 - 751.

[78] D. E. Moncton, G. Shirane, W. Thomlinson, M. Ishikawa, O. Fischer, Coexistence of antiferromagnetism and superconductivity: A neutron diffraction study of DyMo ${ }_{6} \mathrm{~S}_{8}$, Phys. Rev. Lett. 41 (1978) 1133-1136.

[79] C. Majkrzak, G. Shirane, W. Thomlinson, M. Ishikawa, O. Fischer, D. Moncton, A neutron diffraction study of the coexistence of antiferromagnetism and superconductivity in $\mathrm{GdMo}_{6} \mathrm{~S}_{8}$, Solid State Comm. 31 (1979) 773 - 775 .

[80] W. Thomlinson, G. Shirane, D. E. Moncton, M. Ishikawa, Ø. Fischer, Magnetic order in superconducting $\mathrm{TbMo}_{6} \mathrm{~S}_{8}, \mathrm{DyMo}_{6} \mathrm{~S}_{8}, \mathrm{ErMo}_{6} \mathrm{~S}_{8}$, Phys. Rev. B 23 (1981) 4455-4462.

[81] R. McCallum, D. Johnston, R. Shelton, M. Maple, Phase transition in the superconducting state in Gd-rich pseudoternary compounds $\left(\mathrm{La}_{1-x} \mathrm{Gd}_{x}\right)_{1.0} \mathrm{Mo}_{6} \mathrm{Se}_{8}$, Solid State Comm. 24 (1977) $391-395$.

[82] M. Maple, L. Woolf, C. Majkrzak, G. Shirane, W. Thomlinson, D. Moncton, Coexistence of superconductivity and long-range antiferromagnetic order in $\mathrm{Gd}_{1.2} \mathrm{Mo}_{6} \mathrm{Se}_{8}$, Phys. Lett. A 77 (1980) 487 - 489.

[83] Ø. Fischer, M. Ishikawa, M. Pelizzone, A. Treyvaud, Exchange and crystalline field in metallic compounds.coexistence of superconductivity and long range magnetic order, J. Phys. Colloques 40 (1979) 89-94.

[84] M. B. Maple, Superconductivity and magnetic order, J. Phys. Colloques 
39 (1978) 1374-1378

[85] M. Ishikawa, Ø. Fischer, J. Müller, Long range magnetic order in the superconducting state of heavy rare earth molybdenum sulfides and their pseudoternary compounds, J. Phys. Colloques 39 (1978) 1379-1384.

[86] W. A. Fertig, D. C. Johnston, L. E. DeLong, R. W. McCallum, M. B. Maple, B. T. Matthias, Destruction of superconductivity at the onset of long-range magnetic order in the compound $\mathrm{ErRh}_{4} \mathrm{~B}_{4}$, Phys. Rev. Lett. 38 (1977) 987-990.

[87] H. Hamaker, L. Woolf, H. MacKay, Z. Fisk, M. B. Maple, Possible observation of the coexistence of superconductivity and long-range magnetic order in $\mathrm{NdRh}_{4} \mathrm{~B}_{4}$, Solid State Comm. 31 (1979) 139 - 144.

[88] H. Hamaker, L. Woolf, H. MacKay, Z. Fisk, M. B. Maple, Coexistence of superconductivity and antiferromagnetic order in $\mathrm{SmRh}_{4} \mathrm{~B}_{4}$, Solid State Comm. 32 (1979) 289 - 294

[89] D. E. Moncton, D. B. McWhan, J. Eckert, G. Shirane, W. Thomlinson, Neutron scattering study of magnetic ordering in the reentrant superconductor $\mathrm{ErRh}_{4} \mathrm{~B}_{4}$, Phys. Rev. Lett. 39 (1977) 1164-1166.

[90] C. F. Majkrzak, D. E. Cox, G. Shirane, H. A. Mook, H. C. Hamaker, H. B. MacKay, Z. Fisk, M. B. Maple, Neutron-diffraction study of the magnetic ordering in superconducting $\mathrm{NdRh}_{4} \mathrm{~B}_{4}$, Phys. Rev. B 26 (1982) 245-249.

[91] H. Hamaker, H. MacKay, M. Torikachvili, L. Woolf, M. B. Maple, W. Odoni, H. Ott, Observation of the coexistence of superconductivity and long-range magnetic order in $\mathrm{TmRh}_{4} \mathrm{~B}_{4}$, J. Low Temp. Phys. 44 (1981) 553-568

[92] C. F. Majkrzak, S. K. Satija, G. Shirane, H. C. Hamaker, Z. Fisk, M. B. Maple, Neutron-diffraction study of the magnetic ordering in superconducting $\mathrm{TmRh}_{4} \mathrm{~B}_{4}$, Phys. Rev. B 27 (1983) 2889-2893.

[93] L. Woolf, D. C. Johnston, H. MacKay, R. McCallum, M. B. Maple, Superconducting and normal state properties of $\mathrm{ErRh}_{4} \mathrm{~B}_{4}$ and $\mathrm{LuRh}_{4} \mathrm{~B}_{4}$, J. Low Temp. Phys. 35 (1979) 651-669.

[94] K. Kumagai, T. Ooyama, H. Nakajima, M. Shimotomai, Superconducting and magnetic properties of $\mathrm{CeRh}_{4} \mathrm{~B}_{4}$ and $\mathrm{PrRh}_{4} \mathrm{~B}_{4}$, Physica $\mathrm{B}+\mathrm{C}$ 148 (1987) 133 - 136.

[95] R. Chevrel, Thése de doctorat d'état, Ph.D. thesis, University of Rennes, France (1974)

[96] R. Chevrel and M. Sergent, Chemistry and structure of ternary molybdenum chalcogenides, in: $\emptyset$. Fischer, M. B. Maple (Eds.), Superconductivity in Ternary Compounds 1, Springer-Verlag, Berlin Heidelberg New York, 1982, pp. 25-86.

[97] J. M. Vandenberg, B. T. Matthias, Crystallography of new ternary borides, Proc. National Acad. Sci. 74 (1977) 1336-1337.

[98] M. A. Ruderman, C. Kittel, Indirect exchange coupling of nuclear magnetic moments by conduction electrons, Phys. Rev. 96 (1954) 99-102.

[99] T. Kasuya, A theory of metallic ferro- and antiferromagnetism on Zener's model, Prog. Theor. Phys. 16 (1956) 45-57.

[100] K. Yosida, Magnetic properties of Cu-Mn alloys, Phys. Rev. 106 (1957) 893-898.

[101] M. B. Maple, H. C. Hamaker, L. D. Woolf, H. B. MacKay, Z. Fisk, W. Odoni, H. R. Ott, Superconductivity, Long-Range Magnetic Order, and Crystal Field Effects in RERh ${ }_{4} B_{4}$, in: J. E. Crow, R. P. Guertin, T. W. Milhalisin (Eds.), Crystalline Electric Field and Structural Effects in $f$-Electron Systems, Plenum, New York, 1980, pp. 533-546.

[102] Ø. Fischer, M. B. Maple, Superconducting ternary compounds: Prospects and perspectives, in: $\varnothing$. Fischer, M. B. Maple (Eds.), Superconductivity in Ternary Compounds 1, Springer-Verlag, Berlin Heidelberg New York, 1982, pp. 1-23.

[103] J. M. Vandenberg, B. T. Matthias, Clustering hypothesis of some hightemperature superconductors, Science 198 (1977) 194-196.

104] T. Jarlborg, A. J. Freeman, T. J. Watson-Yang, Electronic structure an reentrant magnetism in superconducting $\mathrm{ErRh}_{4} \mathrm{~B}_{4}$, Phys. Rev. Lett. 39 (1977) 1032-1034

[105] M. B. Maple, H. C. Hamaker, D. C. Johnston, H. B. Mackay, L. D. Woolf, Interaction between superconductivity and magnetism in the pseudoternary system $\left(\mathrm{Lu}_{1-x} \mathrm{Ho}_{x}\right) \mathrm{Rh}_{4} \mathrm{~B}_{4}$, J. Less Common Metals 62 (1978) $251-263$.

[106] M. Ishikawa, Ø. Fischer, Destruction of superconductivity by magnetic ordering in $\mathrm{Ho}_{1.2} \mathrm{Mo}_{6} \mathrm{~S}_{8}$, Solid State Comm. 23 (1977) 37 - 39

[107] L. D. Woolf, M. Tovar, H. C. Hamaker, M. B. Maple, Low-temperature heat capacity and ac magnetic susceptivility of the re-entrant ferromagnetic superconductor $\mathrm{Ho}_{1.2} \mathrm{Mo}_{6} \mathrm{~S}_{8}$, Phys. Lett. A 71 (1979) 137 - 139.
[108] J. Lynn, D. Moncton, W. Thomlinson, G. Shirane, R. Shelton, Direct observation of long range ferromagnetic order in the reentrant superconductor $\mathrm{HoMo}_{6} \mathrm{~S}_{8}$, Solid State Comm. 26 (1978) 493 - 496.

[109] Ø. Fischer, M. B. Maple, Magnetic superconductors, in: M. B. Maple, $\varnothing$. Fischer (Eds.), Superconductivity in Ternary Compounds 2, SpringerVerlag, Berlin Heidelberg New York, 1982, pp. 1-10.

[110] D. E. Moncton, D. B. McWhan, P. H. Schmidt, G. Shirane, W. Thomlinson, M. B. Maple, H. B. MacKay, L. D. Woolf, Z. Fisk, D. C. Johnston, Oscillatory magnetic fluctuations near the superconductor-toferromagnet transition in $\mathrm{ErRh}_{4} \mathrm{~B}_{4}$, Phys. Rev. Lett. 45 (1980) 2060 2063.

[111] J. W. Lynn, G. Shirane, W. Thomlinson, R. N. Shelton, D. E. Moncton, Magnetic properties of the reentrant ferromagnetic superconductor $\mathrm{HoMo}_{6} \mathrm{~S}_{8}$, Phys. Rev. B 24 (1981) 3817-3829.

[112] S. K. Sinha, G. W. Crabtree, D. G. Hinks, H. Mook, Study of coexistence of ferromagnetism and superconductivity in single-crystal $\mathrm{ErRh}_{4} \mathrm{~B}_{4}$, Phys. Rev. Lett. 48 (1982) 950-953.

[113] W. Baltensperger, S. Strässler, Superconductivity in antiferromagnets, Physik der Kondensierten Materie 1 (1963) 20-26.

[114] M. Ishikawa, Ø. Fischer, J. Muller, Superconductivity and magnetism in (RE) $\mathrm{Mo}_{6} \mathrm{X}_{8}$ type compounds, in: M. B. Maple, Ø. Fischer (Eds.), Superconductivity in Ternary Compounds 2, Springer-Verlag, Berlin Heidelberg New York, 1982, pp. 143-165.

[115] H. R. Ott, A. Campbell, H. Rudigier, H. Hamaker, M. B. Maple, Superconducting properties of $\mathrm{LuRh}_{4} \mathrm{~B}_{4}$, Physica B + C 108 (1981) $751-$ 752.

[116] H. R. Ott, H. R. Fertig, D. C. Johnston, M. B. Maple, B. T. Matthias, Superconducting and magnetic properties of $\mathrm{ErRh}_{4} \mathrm{~B}_{4}$, J. Low Temp. Phys. 33 (1978) 159-174.

[117] P. Fulde, K. Maki, Theory of superconductors containing magnetic impurities, Phys. Rev. 141 (1966) 275-280.

[118] K. Machida, Superconductivity of ternary rare earth compounds IV. Coexistence of antiferromagnetism and superconductivity, J. Low Temp. Phys. 37 (1979) 583-594.

[119] P. Fulde and J. Keller, Theory of magnetic superconductors, in: M. B. Maple, Ø. Fischer (Eds.), Superconductivity in Ternary Compounds 2, Springer-Verlag, Berlin Heidelberg New York, 1982, pp. 249-294.

[120] D. C. Johnston, W. A. Fertig, M. B. Maple, B. T. Matthias, Re-entrant superconductivity and magnetic ordering in the pseudoternary system $\mathrm{Er}_{1-x} \mathrm{Ho}_{x} \mathrm{Rh}_{4} \mathrm{~B}_{4}$, Solid State Comm. 26 (1978) 141 - 144.

[121] H. A. Mook, O. A. Pringle, S. Kawarazaki, S. K. Sinha, G. W. Crabtree, D. G. Hinks, M. B. Maple, Z. Fisk, D. C. Johnston, L. D. Woolf, H. C. Hamaker, Neutron Scattering Studies of the Magnetic Ordering in Ternary Rare Earth Compounds, in: W. Buckel, W. Weber (Eds.), Superconductivity in $d$ - and $f$-Band Metals, KGK, Karlsruhe, 1982, p. 201.

[122] K. N. Yang, S. E. Lambert, H. C. Hamaker, M. B. Maple, H. A. Mook, H. C. Ku, Low Temperature Investigation of Magnetic Order and Superconductivity in the Pseudoternary System $\operatorname{Ho}\left(\mathrm{Rh}_{1-x} \mathrm{Ir}_{x}\right)_{4} \mathrm{~B}_{4}$, in: W. Buckel, W. Weber (Eds.), Superconductivity in $d$ - and $f$-Band Metals, KGK, Karlsruhe, 1982, p. 217.

[123] H. Ku, H. Braun, F. Acker, Antiferromagnetism in $\mathrm{RE}\left(\mathrm{Rh}_{1-x} \mathrm{Ir}_{x}\right)_{4} \mathrm{~B}_{4}$ compounds, Physica B + C 108 (1981) 1231 - 1232.

[124] S. E. Lambert, M. B. Maple, O. A. Pringle, H. A. Mook, Timedependent magnetic structures of the superconducting mixed ternary system Ho(Rh $\left.\operatorname{Rh}_{1-x} \mathrm{Ir}_{x}\right)_{4} \mathrm{~B}_{4}$, Phys. Rev. B 32 (1985) 2902-2908.

[125] M. Maple, Experiments on magnetically-ordered superconductors, J. Magn. Magn. Mater. 31 - 34 (1983) 479 - 483.

[126] L. Woolf, D. Johnston, H. Mook, W. Koehler, M. Maple, Z. Fisk, Interpretation of specific heat and spontaneous magnetization anomalies at the re-entrant superconducting-ferromagnetic transition in $\left(\mathrm{Ho}_{0.6} \mathrm{Er}_{0.4}\right) \mathrm{Rh}_{4} \mathrm{~B}_{4}$, Physica B+C 109 -110 (1982) 2045 - 2047.

[127] H. Ku, F. Acker, B. Matthias, Superconductivity and magnetic ordering in the pseudoternary $\operatorname{Ho}\left(\operatorname{Ir}_{x} \mathrm{Rh}_{1-x}\right)_{4} \mathrm{~B}_{4}$ system, Phys. Lett. A 76 (1980) $399-402$.

[128] L. Woolf, S. Lambert, M. Maple, F. Acker, H. Ku, W. Odoni, H. Ott, Investigation of the occurrence of superconductivity below the antiferromagnetic transition in $\mathrm{Ho}\left(\mathrm{Ir}_{0.7} \mathrm{Rh}_{0.3}\right)_{4} \mathrm{~B}_{4}$, J. Low Temp. Phys. 51 (1983) 117-134.

[129] H. Hamaker, H. Ku, M. Maple, H. Mook, Neutron diffraction study of $\mathrm{Ho}\left(\mathrm{Rh}_{0.3} \operatorname{Ir}_{0.7}\right)_{4} \mathrm{~B}_{4}$, Solid State Comm. 43 (1982) 455 - 459.

[130] Ø. Fischer, M. B. Maple (Eds.), Superconductivity in Ternary Com- 
pounds 1, Springer-Verlag, Berlin Heidelberg New York, 1982.

[131] Ø. Fischer, M. B. Maple (Eds.), Superconductivity in Ternary Compounds 2, Springer-Verlag, Berlin Heidelberg New York, 1982.

[132] V. Jaccarino, M. Peter, Ultra-high-field superconductivity, Phys. Rev. Lett. 9 (1962) 290-292.

[133] N. R. Werthamer, E. Helfand, P. C. Hohenberg, Temperature and purity dependence of the superconducting critical field, $H_{c 2}$. III. electron spin and spin-orbit effects, Phys. Rev. 147 (1966) 295-302.

[134] E. Helfand, N. R. Werthamer, Temperature and purity dependence of the superconducting critical field, $H_{c 2}$. II, Phys. Rev. 147 (1966) 288-294

[135] K. Maki, Effect of Pauli paramagnetism on magnetic properties of highfield superconductors, Phys. Rev. 148 (1966) 362-369.

[136] M. B. Maple, Induction of superconductivity by applied magnetic fields, Nature 315 (1985) 95-96.

[137] H. W. Meul, C. Rossel, M. Decroux, Ø. Fischer, G. Remenyi, A. Briggs, Observation of magnetic-field-induced superconductivity, Phys. Rev. Lett. 53 (1984) 497-500.

[138] M. Torikachvili, M. Maple, Enhancement of the upper critical magnetic field in $\mathrm{La}_{1.2-x} \mathrm{Eu}_{x} \mathrm{Mo}_{6} \mathrm{~S}_{8}$ compounds, Solid State Comm. 40 (1981) 1 -5 .

[139] M. S. Torikachvili, J. Beille, S. E. Lambert, M. B. Maple, Superconductivity and Magnetic Interactions in $\mathrm{Yb}_{1.2-x} \mathrm{Eu}_{x} \mathrm{Mo}_{6} \mathrm{~S}_{8}$, in: W. Buckel, W. Weber (Eds.), Superconductivity in $d$ - and $f$-Band Metals, KGK, Karlsruhe, 1982, p. 217.

[140] M. Decroux, S. E. Lambert, M. S. Torikachvili, M. B. Maple, R. P. Guertin, L. D. Woolf, R. Baillif, Observation of bulk superconductivity in $\mathrm{EuMo}_{6} \mathrm{~S}_{8}$, Phys. Rev. Lett. 52 (1984) 1563.

[141] M. Decroux, S. Lambert, M. Maple, R. Guertin, Normal and superconducting properties of $\mathrm{EuMo}_{6} \mathrm{~S}_{8}$ in high magnetic fields, J. Low Temp. Phys. 73 (1988) 283-303.

[142] M. Decroux, M. S. Torikachvili, M. B. Maple, R. Baillif, Ø. Fischer, J. Muller, Experimental evidence for bulk superconductive behavior of EuMo $_{6} \mathrm{~S}_{8}$ under pressure, Phys. Rev. B 28 (1983) 6270-6276.

[143] J. Sarrao, M. de Andrade, J. Herrmann, S. Han, Z. Fisk, M. Maple, R. Cava, Superconductivity to $21 \mathrm{~K}$ in intermetallic thorium-based boride carbides, Physica C: Superconductivity 229 (1994) $65-69$

[144] L. C. Gupta, Superconductivity and magnetism and their interplay in quaternary borocarbides $\mathrm{RENi}_{2} \mathrm{~B}_{2} \mathrm{C}$, Advances in Physics 55 (2006) 691-798.

[145] K.-H. Müller, V. Narozhnyi (Eds.), Proceedings of the NATO Advanced Research Workshop (ARW) on Rare Earth Transition Metal Borocarbides (Nitrides): Superconducting, Magnetic and Normal State Properties, Kluwer Academic Publishers, The Netherlands, 2000.

[146] Z. Hossain, S. Dhar, R. Nagarajan, L. Gupta, C. Godart, R. Vijayaraghavan, Magnetic properties of $\mathrm{RNi}_{2} \mathrm{~B}_{2} \mathrm{C}(\mathrm{R}=\mathrm{Pr}, \mathrm{Sm}$, Dy and $\mathrm{Tb})$, IEEE Trans. Magn. 31 (1995) 4133-4135.

[147] B. K. Cho, P. C. Canfield, D. C. Johnston, Onset of superconductivity in the antiferromagnetically ordered state of single-crystal $\mathrm{DyNi}_{2} \mathrm{~B}_{2} \mathrm{C}$, Phys. Rev. B 52 (1995) R3844-R3847.

[148] C. V. Tomy, M. R. Lees, L. Afalfiz, G. Balakrishnan, D. M. Paul, Superconductivity and magnetism in $\mathrm{DyNi}_{2} \mathrm{~B}_{2} \mathrm{C}$ single crystals, Phys. Rev. B 52 (1995) 9186-9189.

[149] Z. Hossain, L. Gupta, R. Nagarajan, S. Dhar, C. Godart, R. Vijayaraghavan, Observation of superconductivity in polycrystalline dyni ${ }_{2} b_{2} c$, Physica B (1996) 99 - 101

[150] H. Eisaki, H. Takagi, R. J. Cava, B. Batlogg, J. J. Krajewski, W. F. Peck, K. Mizuhashi, J. O. Lee, S. Uchida, Competition between magnetism and superconductivity in rare-earth nickel boride carbides, Phys. Rev. B 50 (1994) 647-650.

[151] B. K. Cho, P. C. Canfield, L. L. Miller, D. C. Johnston, W. P. Beyermann, A. Yatskar, Magnetism and superconductivity in single-crystal $\mathrm{ErNi}_{2} \mathrm{~B}_{2} \mathrm{C}$, Phys. Rev. B 52 (1995) 3684-3695.

[152] C. Godart, L. Gupta, R. Nagarajan, S. Dhar, H. Noel, M. Potel, C. Mazumdar, Z. Hossain, C. Levy-Clement, G. Schiffmacher, B. Padalia, R. Vijayaraghavan, Structural, superconducting, and magnetic properties of yni $b_{2} c$ and erni ${ }_{2} b_{2} c$, Phys. Rev. B 51 (1995) 489496.

[153] R. Movshovich, M. Hundley, J. Thompson, P. Canfield, B. Cho, A. Chubukov, Specific heat of single-crystal $\mathrm{YNi}_{2} \mathrm{~B}_{2} \mathrm{C}$ and $\mathrm{TmNi}_{2} \mathrm{~B}_{2} \mathrm{C}$ superconductors, Physica C 227 (1994) $381-386$.

[154] B. K. Cho, M. Xu, P. C. Canfield, L. L. Miller, D. C. Johnston, Magnetic and superconducting properties of single-crystal $\mathrm{TmNi}_{2} \mathrm{~B}_{2} \mathrm{C}$, Phys. Rev. B 52 (1995) 3676-3683.

[155] B. K. Cho, P. C. Canfield, D. C. Johnston, Breakdown of de Gennes scaling in $\mathrm{R}_{1-x} \mathrm{R}^{\prime}{ }_{x} \mathrm{Ni}_{2} \mathrm{~B}_{2} \mathrm{C}$ compounds, Phys. Rev. Lett. 77 (1996) 163 166.

[156] Ø. Fischer, Magnetic Superconductors, in: K. H. J. Buschow, E. P. Wohlfarth (Eds.), Ferromagnetic Materials, Vol. 5, Science Publishers BV, Amsterdam, 1990, p. 465.

[157] J. W. Lynn, S. Skanthakumar, Q. Huang, S. K. Sinha, Z. Hossain, L. C. Gupta, R. Nagarajan, C. Godart, Magnetic order and crystal structure in the superconducting $R \mathrm{Ni}_{2} \mathrm{~B}_{2} \mathrm{C}$ materials, Phys. Rev. B 55 (1997) 65846598.

[158] T. V. Ramakrishnan, C. M. Varma, Pairbreaking in superconductors near and below antiferromagnetic transitions, Phys. Rev. B 24 (1981) 137143 . 